\title{
THE DIRAC EQUATION IN KERR-NEWMAN-ADS BLACK HOLE BACKGROUND
}

\author{
FRANCESCO BELGIORNO AND SERGIO L. CACCIATORI
}

\begin{abstract}
We consider the Dirac equation on the Kerr-Newman-AdS black hole background. We first perform the variable separation for the Dirac equation and define the Hamiltonian operator $\hat{H}$. Then we show that for a massive Dirac field with mass $\mu \geq \frac{1}{2 l}$ essential selfadjointness of $\hat{H}$ on $C_{0}^{\infty}\left(\left(r_{+}, \infty\right) \times S^{2}\right)^{4}$ is obtained even in presence of the boundary-like behavior of infinity in an asymptotically AdS black hole background. Furthermore qualitative spectral properties of the Hamiltonian are taken into account and in agreement with the existing results concerning the case of stationary axi-symmetric asymptotically flat black holes we infer the absence of time-periodic and normalizable solutions of the Dirac equation around the black hole in the non-extremal case.
\end{abstract}

\section{INTRODUCTION}

Black holes play a very important role in many aspects of theoretical physics, starting from the fact that they provide nontrivial exact solutions of Einstein equations, going through their thermodynamical properties and ending up with their role of "hydrogen atoms of quantum gravity" [1. Their relevance is fundamental also beyond the theoretical aspects. The uniqueness and the no hair theorems (2]) give strong restrictions on the possible signatures one could look for in astrophysical observations. For real situations one needs to take account the fact that black holes are not simply vacuum solutions. Accretion disks in active galactic nuclei constitute one of the most studied questions about "real" black hole physics. Another interesting problem which concerns rotating charged black holes is their electrical shielding by a charged dust; its solution could give rise to new astrophysics 1 Moreover, other signatures could arise from quantum effects like black hole discharge (see for example [3, 4, 5]) or angular momentum loss.

Among the theoretical models which can provide a deeper understanding of the mathematical properties of the field equations for the matter fields living on the given black hole background and also of thermodynamical contributions of the matter fields to black hole thermodynamics the asymptotically AdS case is interesting under many respects, both for the well-known relevance of the AdS backgrounds in the AdS-CFT conjecture and in supergravity, and for the peculiar thermodynamical properties of AdS black holes, for which the canonical ensemble is well-defined [6]. In order to avoid the presence of closed time-like curves, one has to take into account the universal covering of such an AdS black hole background, which is not globally hyperbolic [6]. Notwithstanding, physics can still be uniquely defined if essential selfadjointness properties are obtained at least for suitable sets of the field parameters like e.g. the mass (cf. [7]). Herein, we limit ourselves to consider the specific problem of a spin $\frac{1}{2}$ massive charged Dirac field on the background of an Anti-de Sitter charged rotating black hole. Our aim is twofold: on one hand, we pursue the variable separation following [8] and the analysis of the Hamiltonian description in the given background, generalizing the known results for the asymptotically flat Kerr-Newman solution [9]; on the other hand, we consider the problem of essential selfadjointness of the Hamiltonian $\hat{H}$ on $C_{0}^{\infty}\left(\left(r_{+}, \infty\right) \times S^{2}\right)^{4}$ for the Dirac field on a Kerr-Newman-AdS black hole background in presence also of a magnetic charge. This second task is nontrivial, requiring many technical steps in order to be settled. The main difficulty arises from the fact that even if for the Hamiltonian version of the Dirac equation a Chandrasekhar-like ansatz for variable separation is available, one cannot obtain a full reduction of the Hamiltonian into an orthogonal sum of partial wave operators involving only the radial variable, and this has to be attributed to the black hole rotation which allows only axial symmetry. Nevertheless, essential selfadjointness of $\hat{H}$ on $C_{0}^{\infty}\left(\left(r_{+}, \infty\right) \times S^{2}\right)^{4}$ is shown to be equivalent to the essential selfadjointness of another Hamiltonian $\hat{H}_{0}$ on $C_{0}^{\infty}\left(\left(r_{+}, \infty\right) \times S^{2}\right)^{4}$ which is defined in a different (but unitarily equivalent) Hilbert space, and a complete reduction into an orthogonal sum of partial wave operators involving only the radial variable for $\hat{H}_{0}$ is shown to

\footnotetext{
${ }^{1}$ This problem was pointed out to us by Aldo Treves.
} 
be available. This turns out to be useful also for the analysis of qualitative spectral properties of $\hat{H}$. It is worth mentioning that the aforementioned problem associated with variable separation in Chandrasekhar ansatz and the occurrence of two Hilbert spaces in the analysis of the Dirac equation have been already pointed out in 9 for the case of the Dirac equation on a black hole background of the Kerr-Newman family, which has been considered in several studies [10, 11, 12, 13, 14, 15, (see also [16]); the above part of our analysis can be of interest also for that case. Moreover, our analysis points out some relevant differences to be related to the AdS background considered herein, and also introduces some interesting analysis to be associated with a magnetically charged black hole: we find the Dirac charge quantization as a condition ensuring essential selfadjointness for the Hamiltonian operator $\hat{H}$ on $C_{0}^{\infty}\left(\left(r_{+}, \infty\right) \times S^{2}\right)^{4}$. Furthermore, as a corollary of our qualitative spectral analysis, we can also conclude that in the non-extremal case there is no normalizable time-periodic solution of the Dirac equation, in agreement with the result for the Kerr-Newman case [11].

The plan of the paper is the following. In section 2 we describe the background geometry. In section 3 we consider the equation for the Dirac field. Section 4 is devoted to the study of the Hamiltonian formulation. In section 5 the essential selfadjointness of the Hamiltonian is analyzed, and in section 6 some spectral properties are deduced. In particular, we can conclude that in the non-extremal case the point spectrum is empty. Three appendices complete our analysis.

\section{The Kerr-Newman-AdS solution.}

We will give a short description of the background geometry underlying our problem. It arises as follows. One first solves the Einstein-Maxwell equations with a cosmological constant, and next adds a Dirac field minimally coupled to the electromagnetic field. The Einstein-Maxwell action is

$$
S\left[g_{\mu \nu}, A_{\rho}\right]=-\frac{1}{16 \pi} \int(R-2 \Lambda) \sqrt{-\operatorname{det} g} d^{4} x-\frac{1}{16 \pi} \int \frac{1}{4} F_{\mu \nu} F^{\mu \nu} \sqrt{-\operatorname{det} g} d^{4} x,
$$

where $\Lambda=-\frac{3}{l^{2}}$ is the cosmological constant, $R$ the scalar curvature and $F_{\mu \nu}$ the field strength associated to the potential 1-form $A$ :

$$
\begin{aligned}
& F=d A, \quad F_{\mu \nu}=\partial_{\mu} A_{\nu}-\partial_{\nu} A_{\mu} \\
& R=g^{\mu \nu} R_{\mu \nu}, \quad R_{\mu \nu}=\partial_{\rho} \Gamma_{\mu \nu}^{\rho}-\partial_{\nu} \Gamma_{\mu \rho}^{\rho}+\Gamma_{\mu \nu}^{\sigma} \Gamma_{\sigma \rho}^{\rho}-\Gamma_{\mu \rho}^{\sigma} \Gamma_{\sigma \nu}^{\rho} \\
& \Gamma_{\nu \rho}^{\mu}=\frac{1}{2} g^{\mu \sigma}\left(\partial_{\nu} g_{\sigma \rho}+\partial_{\rho} g_{\sigma \nu}-\partial_{\sigma} g_{\nu \rho}\right)
\end{aligned}
$$

The equations of motion are

$$
\begin{aligned}
& R_{\mu \nu}-\frac{1}{2}(R-2 \Lambda) g_{\mu \nu}=-2\left(F_{\mu}{ }^{\rho} F_{\nu \rho}-\frac{1}{4} g_{\mu \nu} F_{\rho \sigma} F^{\rho \sigma}\right), \\
& \partial_{\mu}\left(\sqrt{-\operatorname{det} g} F^{\mu \nu}\right)=0 .
\end{aligned}
$$

With respect to a set of vierbein one forms

$$
e^{i}=e_{\mu}^{i} d x^{\mu}, \quad i=0,1,2,3,
$$

we have

$$
d s^{2}=g=\eta_{i j} e^{i} \otimes e^{j}, \quad g_{\mu \nu}=\eta_{i j} e_{\mu}^{i} e_{\nu}^{j},
$$

where $\eta=\operatorname{diag}(-1,1,1,1)$ is the usual flat Minkowski metric, so that, as usual, we define the $s o(1,3)$ valued spin connection one forms $\omega_{j}^{i}$ such that

$$
d e^{i}+\omega_{j}^{i} \wedge e^{j}=0 .
$$

We will consider the following background solution.

The metric is

$$
d s^{2}=-\frac{\Delta_{r}}{\rho^{2}}\left[d t-\frac{a \sin ^{2} \theta}{\Xi} d \phi\right]^{2}+\frac{\rho^{2}}{\Delta_{r}} d r^{2}+\frac{\rho^{2}}{\Delta_{\theta}} d \theta^{2}+\Delta_{\theta} \frac{\sin ^{2} \theta}{\rho^{2}}\left[a d t-\frac{r^{2}+a^{2}}{\Xi} d \phi\right]^{2},
$$


where

$$
\begin{array}{ll}
\rho^{2}=r^{2}+a^{2} \cos ^{2} \theta, & \Xi=1-\frac{a^{2}}{l^{2}}, \quad \Delta_{r}=\left(r^{2}+a^{2}\right)\left(1+\frac{r^{2}}{l^{2}}\right)-2 m r+z^{2}, \\
\Delta_{\theta}=1-\frac{a^{2}}{l^{2}} \cos ^{2} \theta, \quad z^{2}=q_{e}^{2}+q_{m}^{2},
\end{array}
$$

and the electromagnetic potential and field strength are

$$
\begin{aligned}
A= & -\frac{q_{e} r}{\rho \sqrt{\Delta_{r}}} e^{0}-\frac{q_{m} \cos \theta}{\rho \sqrt{\Delta_{\theta}} \sin \theta} e^{1}, \\
F= & -\frac{1}{\rho^{4}}\left[q_{e}\left(r^{2}-a^{2} \cos ^{2} \theta\right)+2 q_{m} r a \cos \theta\right] e^{0} \wedge e^{2} \\
& +\frac{1}{\rho^{4}}\left[q_{m}\left(r^{2}-a^{2} \cos ^{2} \theta\right)-2 q_{e} r a \cos \theta\right] e^{3} \wedge e^{1},
\end{aligned}
$$

where we introduced the vierbein

$$
\begin{aligned}
e^{0} & =\frac{\sqrt{\Delta_{r}}}{\rho}\left(d t-\frac{a \sin ^{2} \theta}{\Xi} d \phi\right), \\
e^{1} & =\frac{\sqrt{\Delta_{\theta}} \sin \theta}{\rho}\left(a d t-\frac{r^{2}+a^{2}}{\Xi} d \phi\right), \\
e^{2} & =\frac{\rho}{\sqrt{\Delta_{r}}} d r, \\
e^{3} & =\frac{\rho}{\sqrt{\Delta_{\theta}}} d \theta .
\end{aligned}
$$

The parameters $m, a, q_{e}, q_{t}$ are related to the mass, angular momentum, electric and magnetic charge by the Komar integrals (see [17])

$$
M=\frac{m}{\Xi^{2}}, \quad J=\frac{a m}{\Xi^{2}}, \quad Q_{e}=\frac{q_{e}}{\Xi}, \quad Q_{m}=\frac{q_{m}}{\Xi} .
$$

We are interested in the case when an event horizon (corresponding to $\Delta_{\theta}=0$ ) appears, that is for $m \geq m_{e x t}$,

$$
\begin{aligned}
m_{\text {ext }}= & \frac{l}{3 \sqrt{6}}\left(\sqrt{\left(1+\frac{a^{2}}{l^{2}}\right)^{2}+\frac{12}{l^{2}}\left(a^{2}+z^{2}\right)}+2 \frac{a^{2}}{l^{2}}+2\right) \\
& \times\left(\sqrt{\left(1+\frac{a^{2}}{l^{2}}\right)^{2}+\frac{12}{l^{2}}\left(a^{2}+z^{2}\right)}-\frac{a^{2}}{l^{2}}-1\right)^{\frac{1}{2}} .
\end{aligned}
$$




\section{The Dirac EQUATion.}

The Dirac equation for a charged massive particle of mass $\mu$ and electric charge $e$ is

$$
\left(i \gamma^{\mu} D_{\mu}-\mu\right) \psi=0
$$

where $D$ is the Koszul connection on the bundle $S \otimes U(1), S$ being the spin bundle over the AdS-Kerr-Newman manifold, that is

$$
D_{\mu}=\partial_{\mu}+\frac{1}{4} \omega_{\mu}^{i j} \Gamma_{i} \Gamma_{j}+i e A_{\mu}
$$

Here $\omega^{i j}=\omega^{i}{ }_{k} \eta^{k j}$ are the spin connection one forms associated to a vierbein $v^{i}$, such that $d s^{2}=\eta_{i j} v^{i} \otimes v^{j}, \eta$ being the usual Minkowski metric. $\gamma_{\mu}$ are the local Dirac matrices, related to the point independent Minkowskian Dirac matrices $\Gamma_{i}$ by the relations $\gamma_{\mu}=v_{\mu}^{i} \Gamma_{i}$.

Here we use the representation

$$
\Gamma^{0}=\left(\begin{array}{cc}
\mathbb{O} & -\mathbb{I} \\
-\mathbb{I} & \mathbb{O}
\end{array}\right), \quad \vec{\Gamma}=\left(\begin{array}{cc}
\mathbb{O} & -\vec{\sigma} \\
\vec{\sigma} & \mathbb{O}
\end{array}\right)
$$

where

$$
\mathbb{O}=\left(\begin{array}{ll}
0 & 0 \\
0 & 0
\end{array}\right), \quad \mathbb{I}=\left(\begin{array}{ll}
1 & 0 \\
0 & 1
\end{array}\right)
$$

and $\vec{\sigma}$ are the usual Pauli matrices

$$
\sigma_{1}=\left(\begin{array}{cc}
0 & 1 \\
1 & 0
\end{array}\right), \quad \sigma_{2}=\left(\begin{array}{cc}
0 & -i \\
i & 0
\end{array}\right), \quad \sigma_{3}=\left(\begin{array}{cc}
1 & 0 \\
0 & -1
\end{array}\right) .
$$

Thus

$$
\gamma_{\mu} \gamma_{\nu}+\gamma_{\nu} \gamma_{\mu}=-2 g_{\mu \nu} .
$$

We can now separate variables following the general results of 8 . Let us introduce the null Newman-Penrose (symmetric) frame

$$
\begin{aligned}
\theta^{1} & =\frac{1}{\sqrt{2}}|Z(r, \theta)|^{\frac{1}{2}}\left[\frac{W(r)}{Z(r, \theta)}\left(d t+\frac{a \sin ^{2} \theta}{\Xi} d \phi\right)+\frac{d r}{W(r)}\right] \\
\theta^{2} & =\frac{1}{\sqrt{2}}|Z(r, \theta)|^{\frac{1}{2}}\left[\frac{W(r)}{Z(r, \theta)}\left(d t+\frac{a \sin ^{2} \theta}{\Xi} d \phi\right)-\frac{d r}{W(r)}\right] \\
\theta^{3} & =\frac{1}{\sqrt{2}}|Z(r, \theta)|^{\frac{1}{2}}\left[\frac{X(\theta)}{Z(r, \theta)}\left(a d t-\frac{r^{2}+a^{2}}{\Xi} d \phi\right)+i \frac{\sin \theta d \theta}{X(\theta)}\right], \\
\theta^{4} & =\bar{\theta}^{3}
\end{aligned}
$$

where

$$
Z(r, \theta)=\frac{r^{2}+a^{2} \cos ^{2} \theta}{\Xi}, \quad W(r)=\frac{\sqrt{\Delta_{r}}}{\Xi^{\frac{1}{2}}}, \quad X(\theta)=\frac{\sqrt{\Delta_{\theta}} \sin \theta}{\Xi^{\frac{1}{2}}},
$$

so that

$$
d s^{2}=-2\left(\theta^{1} \theta^{2}-\theta^{3} \theta^{4}\right)
$$

and

$$
A=-\frac{1}{\sqrt{2|Z(r, \theta)|}}\left[\frac{H(r)}{W(r)}\left(\theta^{1}+\theta^{2}\right)+\frac{G(\theta)}{X(\theta)}\left(\theta^{3}+\theta^{4}\right)\right]
$$

with

$$
H(r)=Q_{e} r, \quad G(\theta)=Q_{m} \cos \theta .
$$


Note that

$$
\begin{aligned}
e^{0} & =\frac{1}{\sqrt{2}}\left(\theta^{1}+\theta^{2}\right), & e^{1} & =\frac{1}{\sqrt{2}}\left(\theta^{3}+\theta^{4}\right), \\
e^{3} & =\frac{1}{\sqrt{2}}\left(\theta^{1}-\theta^{2}\right), & e^{4} & =\frac{1}{\sqrt{2} i}\left(\theta^{3}-\theta^{4}\right) .
\end{aligned}
$$

The Petrov type D condition ensures the existence of a phase function $\mathcal{B}(r, \theta)$ such that

$$
d \mathcal{B}=\frac{1}{4 Z(r, \theta)}\left(-2 a \frac{\cos \theta}{\Xi} d r-2 a r \frac{\sin \theta}{\Xi} d \theta\right)
$$

which indeed gives

$$
\mathcal{B}(r, \theta)=\frac{i}{4} \log \frac{r-i a \cos \theta}{r+i a \cos \theta} .
$$

Now let us write the Dirac equation as

$$
H_{D} \psi=0 .
$$

Under a transformation $\psi \mapsto S^{-1} \psi$, with

$$
S=Z^{-\frac{1}{4}} \operatorname{diag}\left(e^{i \mathcal{B}}, e^{i \mathcal{B}}, e^{-i \mathcal{B}}, e^{-i \mathcal{B}}\right),
$$

it changes as

$$
S^{-1} H_{D} S\left(S^{-1} \psi\right)=0
$$

If we multiply this equation times

$$
U=i Z^{\frac{1}{2}} \operatorname{diag}\left(e^{2 i \mathcal{B}},-e^{2 i \mathcal{B}},-e^{-2 i \mathcal{B}}, e^{-2 i \mathcal{B}}\right),
$$

and introduce the new wave function

$$
\tilde{\psi}=\left(\Delta_{\theta} \Delta_{r}\right)^{\frac{1}{4}} S^{-1} \psi,
$$

then the Dirac equation takes the form

$$
(\mathcal{R}(r)+\mathcal{A}(\theta)) \tilde{\psi}=0,
$$

where

$$
\begin{aligned}
\mathcal{R} & =\left(\begin{array}{cccc}
i \mu r & 0 & -\sqrt{\Delta_{r}} \mathcal{D}_{+} & 0 \\
0 & -i \mu r & 0 & -\sqrt{\Delta_{r}} \mathcal{D}_{-} \\
-\sqrt{\Delta_{r}} \mathcal{D}_{-} & 0 & -i \mu r & 0 \\
0 & -\sqrt{\Delta_{r}} \mathcal{D}_{+} & 0 & i \mu r
\end{array}\right), \\
\mathcal{A} & =\left(\begin{array}{cccc}
-a \mu \cos \theta & 0 & 0 & -i \sqrt{\Delta_{\theta}} \mathcal{L}_{-} \\
0 & a \mu \cos \theta & -i \sqrt{\Delta_{\theta}} \mathcal{L}_{+} & 0 \\
0 & -i \sqrt{\Delta_{\theta}} \mathcal{L}_{-} & -a \mu \cos \theta & 0 \\
-i \sqrt{\Delta_{\theta}} \mathcal{L}_{+} & 0 & 0 & a \mu \cos \theta
\end{array}\right),
\end{aligned}
$$

and

$$
\begin{aligned}
& \mathcal{D}_{ \pm}=\partial_{r} \pm \frac{1}{\Delta_{r}}\left(\left(r^{2}+a^{2}\right) \partial_{t}-a \Xi \partial_{\phi}+i e q_{e} r\right) \\
& \mathcal{L}_{ \pm}=\partial_{\theta}+\frac{1}{2} \cot \theta \pm \frac{i}{\Delta_{\theta} \sin \theta}\left(\Xi \partial_{\phi}-a \sin ^{2} \theta \partial_{t}+i e q_{m} \cos \theta\right)
\end{aligned}
$$

Separation of variables can then be obtained searching for solutions of the form

$$
\tilde{\psi}(t, \phi, r, \theta)=e^{-i \omega t} e^{-i k \phi}\left(\begin{array}{c}
R_{1}(r) S_{2}(\theta) \\
R_{2}(r) S_{1}(\theta) \\
R_{2}(r) S_{2}(\theta) \\
R_{1}(r) S_{1}(\theta)
\end{array}\right), \quad k \in \mathbb{Z}+\frac{1}{2} .
$$




\section{HAMiltonian Formulation.}

The Hamiltonian for the Dirac equation can be read from (3.24) rewriting it in the form [9]

$$
i \partial_{t} \tilde{\psi}=H \tilde{\psi} \text {. }
$$

Indeed we find

$$
H=\left[\left(1-\frac{\Delta_{r}}{\Delta_{\theta}} \frac{a^{2} \sin ^{2} \theta}{\left(r^{2}+a^{2}\right)^{2}}\right)^{-1}\left(\mathbb{I}_{4}-\frac{\sqrt{\Delta_{r}}}{\sqrt{\Delta_{\theta}}} \frac{a \sin \theta}{r^{2}+a^{2}} B C\right)\right](\tilde{\mathcal{R}}+\tilde{\mathcal{A}}),
$$

where $\mathbb{I}_{4}$ is the $4 \times 4$ identity matrix,

$$
\begin{aligned}
& \tilde{\mathcal{R}}=-\frac{\mu r \sqrt{\Delta_{r}}}{r^{2}+a^{2}}\left(\begin{array}{cccc}
0 & 0 & 1 & 0 \\
0 & 0 & 0 & 1 \\
1 & 0 & 0 & 0 \\
0 & 1 & 0 & 0
\end{array}\right)+\left(\begin{array}{cccc}
\mathcal{E}_{-} & 0 & 0 & 0 \\
0 & -\mathcal{E}_{+} & 0 & 0 \\
0 & 0 & -\mathcal{E}_{+} & 0 \\
0 & 0 & 0 & \mathcal{E}_{-}
\end{array}\right) \\
& \tilde{\mathcal{A}}=\frac{a \mu \cos \theta \sqrt{\Delta_{r}}}{r^{2}+a^{2}}\left(\begin{array}{cccc}
0 & 0 & i & 0 \\
0 & 0 & 0 & i \\
-i & 0 & 0 & 0 \\
0 & -i & 0 & 0
\end{array}\right)+\left(\begin{array}{cccc}
0 & -\mathcal{M}_{-} & 0 & 0 \\
\mathcal{M}_{+} & 0 & 0 & 0 \\
0 & 0 & 0 & \mathcal{M}_{-} \\
0 & 0 & -\mathcal{M}_{+} & 0
\end{array}\right) \\
& \mathcal{E}_{ \pm}=i \frac{\Delta_{r}}{a^{2}+r^{2}}\left[\partial_{r} \mp \frac{a \Xi}{\Delta_{r}} \partial_{\phi} \pm i \frac{e q_{e} r}{\Delta_{r}}\right] \\
& \mathcal{M}_{ \pm}=\frac{\sqrt{\Delta_{r}} \sqrt{\Delta_{\theta}}}{r^{2}+a^{2}}\left[\partial_{\theta}+\frac{1}{2} \cot \theta \pm \frac{i \Xi}{\Delta_{\theta} \sin \theta} \partial_{\phi} \mp \frac{e q_{m} \cot \theta}{\Delta_{\theta}}\right]
\end{aligned}
$$

and

$$
B=\left(\begin{array}{cccc}
0 & 0 & -i & 0 \\
0 & 0 & 0 & i \\
i & 0 & 0 & 0 \\
0 & -i & 0 & 0
\end{array}\right), \quad C=\left(\begin{array}{cccc}
0 & 0 & 0 & i \\
0 & 0 & -i & 0 \\
0 & i & 0 & 0 \\
-i & 0 & 0 & 0
\end{array}\right)
$$

satisfy $[B, C]=0, B^{2}=C^{2}=\mathbb{I}_{4}$. Cf. also 9 for the Kerr-Newman case. We need now to specify the Hilbert space. We do it as follows.

If we foliate spacetime in $t=$ constant slices $\mathcal{S}_{t}$, the metric on any slice (considering the shift vectors) is

$$
d \gamma^{2}=\gamma_{\alpha \beta} d x^{\alpha} d x^{\beta}
$$

where $\alpha=1,2,3$ and

$$
\gamma_{\alpha \beta}=g_{\alpha \beta}-\frac{g_{0 \alpha} g_{0 \beta}}{g_{00}}
$$

and local measure

$$
d \mu_{3}=\sqrt{\operatorname{det} \gamma} d r d \theta d \phi=\frac{\sin \theta}{\Xi} \frac{\rho^{3}}{\sqrt{\Delta_{r}-a^{2} \Delta_{\theta} \sin ^{2} \theta}} d r d \theta d \phi
$$

In particular the four dimensional measure factors as

$$
d \mu_{4}=\sqrt{-g_{00}} d \mu_{3} d t .
$$

The action for a massless uncharged Dirac particle is then

$$
S=\int_{\mathbb{R}} d t \int_{\mathcal{S}_{t}} \sqrt{-g_{00}}{ }^{t} \psi^{*} \Gamma^{0} \gamma^{\mu} D_{\mu} \psi d \mu_{3},
$$

where the star indicates complex conjugation. Here with $\mathcal{S}_{t}$ we mean the range of coordinates parameterizing the region external to the event horizon: $r>r_{+}$, that is $\mathcal{S}_{t}:=\mathcal{S}=\left(r_{+}, \infty\right) \times(0, \pi) \times(0,2 \pi)$. Then, the scalar product between wave functions should be

$$
\langle\psi \mid \chi\rangle=\int_{\mathcal{S}} \sqrt{-g_{00}}{ }^{t} \psi^{*} \Gamma^{0} \gamma^{t} \chi d \mu_{3} .
$$


We can now use (4.10), (3.23) and the relation

$$
\gamma^{2}=e_{0}^{t} \Gamma^{0}+e_{1}^{t} \Gamma^{1}
$$

to express the product in the space of reduced wave functions (i.e. (3.23)):

$$
\langle\tilde{\psi} \mid \tilde{\chi}\rangle=\int_{r_{+}}^{\infty} d r \int_{0}^{\pi} d \theta \int_{0}^{2 \pi} d \phi \frac{r^{2}+a^{2}}{\Delta_{r}} \frac{\sin \theta}{\sqrt{\Delta_{\theta}}} t \tilde{\psi}^{*}\left(\mathbb{I}_{4}+\frac{\sqrt{\Delta_{r}}}{\sqrt{\Delta_{\theta}}} \frac{a \sin \theta}{r^{2}+a^{2}} B C\right) \tilde{\chi},
$$

where we have dropped a factor $\Xi^{-\frac{1}{2}}$. Note that the matrix in the parenthesis is the inverse of the one in the square brackets in (4.2), and coincides with the one introduced in [9] (improved to the AdS case).

We show that the above scalar product is positive definite. With this aim, being \pm 1 the eigenvalues of $B C$, we need to prove that

$$
\eta:=\sup _{r \in\left(r_{+}, \infty\right), \theta \in(0, \pi)} \alpha(r, \theta)<1
$$

where

$$
\alpha(r, \theta)=\frac{\sqrt{\Delta_{r}}}{\sqrt{\Delta_{\theta}}} \frac{a \sin \theta}{r^{2}+a^{2}} .
$$

We can write $\alpha(r, \theta)=\beta(r) \gamma(\theta)$, with

$$
\gamma(\theta)=\frac{\sin \theta}{\sqrt{\Delta_{\theta}}}
$$

Then

$$
\gamma^{\prime}(\theta)=\frac{\cos \theta}{\Delta_{\theta}^{\frac{3}{2}}}\left(1-\frac{a^{2}}{l^{2}}\right)
$$

so that $\gamma$ reaches its maximum at $\theta=\pi / 2$ and

$$
\gamma(\pi / 2)=1
$$

which implies

$$
\alpha(r, \theta) \leq \beta(r) .
$$

Next, from

$$
0=\Delta_{r}\left(r_{+}\right)
$$

we have

$$
z^{2}-2 m r_{+}=-\left(r_{+}^{2}+a^{2}\right)\left(r_{+}^{2}+l^{2}\right) / l^{2}<0,
$$

and then, for $r \geq r_{+}$we have $z^{2}-2 m r<0$. Thus

$$
\beta^{2}(r)=\frac{a^{2} \Delta_{r}}{\left(r^{2}+a^{2}\right)^{2}}=\frac{a^{2}}{l^{2}} \frac{r^{2}+l^{2}}{r^{2}+a^{2}}+a^{2} \frac{z^{2}-2 m r}{\left(r^{2}+a^{2}\right)^{2}} \leq \frac{a^{2}}{l^{2}} \frac{r^{2}+l^{2}}{r^{2}+a^{2}}=h(r) .
$$

Now, the last function is a decreasing function of $r$, so that for $r \geq r_{+}>0$ we have $h(r) \leq h\left(r_{+}\right)<h(0)$, so that

$$
\beta^{2}(r) \leq h\left(r_{+}\right)=\frac{a^{2}}{l^{2}} \frac{r_{+}^{2}+l^{2}}{r_{+}^{2}+a^{2}}<h(0)=1
$$

and

$$
\eta \leq \sqrt{h\left(r_{+}\right)}<1
$$




\section{Essential SElfadjointness OF $\hat{\boldsymbol{H}}$.}

Let us introduce the space of functions $\mathcal{L}^{2}:=\left(L^{2}\left(\left(r_{+}, \infty\right) \times S^{2} ; d \mu\right)\right)^{4}$ with measure

$$
d \mu=\frac{r^{2}+a^{2}}{\Delta_{r}} \frac{\sin \theta}{\sqrt{\Delta_{\theta}}} d r d \theta d \phi
$$

and define $\mathcal{H}_{<>}$as the Hilbert space $\mathcal{L}^{2}$ with the scalar product (4.15). We will also consider a second Hilbert space $\mathcal{H}_{()}$, which is obtained from $\mathcal{L}^{2}$ with the scalar product

$$
(\psi \mid \chi)=\int_{r_{+}}^{\infty} d r \int_{0}^{\pi} d \theta \int_{0}^{2 \pi} d \phi \frac{r^{2}+a^{2}}{\Delta_{r}} \frac{\sin \theta}{\sqrt{\Delta_{\theta}}}{ }^{t} \psi^{*} \chi=\int d \mu^{t} \psi^{*} \chi .
$$

It is straightforward to show that $\|\cdot\|_{<>}$and $\|\cdot\|_{()}$are equivalent norms. It is also useful to introduce $\hat{\Omega}^{2}: \mathcal{L}^{2} \rightarrow \mathcal{L}^{2}$ as the multiplication operator by $\Omega^{2}(r, \theta)$ :

$$
\Omega^{2}(r, \theta):=\mathbb{I}_{4}+\alpha(r, \theta) B C .
$$

Then we have

$$
\langle\psi \mid \chi\rangle=\int d \mu^{t} \psi^{*} \Omega^{2} \chi=\left(\psi \mid \hat{\Omega}^{2} \chi\right) .
$$

We introduce also $\hat{\Omega}^{-2}: \mathcal{L}^{2} \rightarrow \mathcal{L}^{2}$ as the multiplication operator by $\Omega^{-2}$ :

$$
\Omega^{-2}(r, \theta):=\frac{1}{1-\alpha^{2}(r, \theta)}\left(\mathbb{I}_{4}-\alpha(r, \theta) B C\right),
$$

and analogously $\hat{\Omega}, \hat{\Omega}^{-1}$ are defined as operators from $\mathcal{L}^{2}$ to $\mathcal{L}^{2}$ which multiply by $\Omega(r, \theta), \Omega^{-1}(r, \theta)$ respectively, where $\Omega$ and $\Omega^{-1}$ are defined as the principal square root of $\Omega^{2}$ and $\Omega^{-2}$ respectively. The following properties are useful for our subsequent analysis. As matrix-valued functions, both $\Omega^{2}$ and $\Omega^{-2}$ are trivially bounded, and this holds true also for $\Omega$ and $\Omega^{-1} . \hat{\Omega}^{2}, \hat{\Omega}, \hat{\Omega}^{-2}, \hat{\Omega}^{-1}$ are injective and surjective; moreover, as operators from $\mathcal{H}_{()}$ to $\mathcal{H}_{()}$, they are positive, bounded and selfadjoint. Injectivity of $\hat{\Omega}^{2}, \hat{\Omega}^{-2}$ can be proven by direct inspection. Being $\hat{\Omega}^{2}$ injective, also $\hat{\Omega}$ is injective. Surjectivity is also easily deduced. Indeed, being $\hat{\Omega}^{-2}$ defined everywhere, $\hat{\Omega}^{2}$ is also surjective and then $\hat{\Omega}$ is surjective too. The same properties hold true for $\hat{\Omega}^{-2}$ and $\hat{\Omega}^{-1}$. Positivity is easily proven by carrying the matrices $\Omega^{2}, \Omega, \Omega^{-2}, \Omega^{-1}$ into the diagonal form and then by taking into account that $\sup _{r, \theta} \alpha<1$ for $a^{2}<l^{2}$. Analogously also boundedness is proven. Positivity implies selfadjointness.

Let us set $H_{0}:=\tilde{\mathcal{R}}+\tilde{\mathcal{A}}$, which is formally selfadjoint on $\mathcal{H}_{()}$, and define the operator $\hat{H}_{0}$ on $\mathcal{L}^{2}$ with

$$
\begin{aligned}
& D\left(\hat{H}_{0}\right)=C_{0}^{\infty}\left(\left(r_{+}, \infty\right) \times S^{2}\right)^{4}=: \mathcal{D} \\
& \hat{H}_{0} \chi=H_{0} \chi, \quad \chi \in \mathcal{D} .
\end{aligned}
$$

Notice that $\mathcal{D}$ is dense in $\mathcal{H}_{()}$. Let us point out that for the formal differential expression $H$ in (4.2), which is formally selfadjoint on $\mathcal{H}_{<>}$, one can write $H=\Omega^{-2} H_{0}$, Then we define on $\mathcal{L}^{2}$ the differential operator $\hat{H}=\hat{\Omega}^{-2} \hat{H}_{0}$, with

$$
\begin{aligned}
& D(\hat{H})=\mathcal{D} \\
& \hat{H} \chi=H \chi, \quad \chi \in \mathcal{D} .
\end{aligned}
$$

As to the symmetry of $\hat{H}$ on $\mathcal{D} \subset \mathcal{H}_{<>}$, we note that for all $f, g \in \mathcal{D}$ it holds $\langle f \mid \hat{H} g\rangle=\left(f \mid \hat{H}_{0} g\right)$ and $\langle\hat{H} f \mid g\rangle=$ $\left(\hat{H} f \mid \hat{\Omega}^{2} g\right)=\left(\hat{\Omega}^{2} \hat{H} f \mid g\right)=\left(\hat{H}_{0} f \mid g\right)$, and then $\hat{H}$ is symmetric iff $\hat{H}_{0}$ is symmetric on $\mathcal{D} \subset \mathcal{H}_{()}$. Symmetry of $\hat{H}_{0}$ is proven by direct inspection: The only problem could be the integration by parts in $\theta$ and $r$. The $r$ derivatives arise in the scalar product from the terms involving the differential operators $\mathcal{E}_{ \pm}$, and they appear in the following form:

$$
\int \frac{\sin \theta}{\sqrt{\Delta_{\theta}}}{ }^{t} \tilde{\psi}^{*} \operatorname{diag}\left(i \partial_{r},-i \partial_{r},-i \partial_{r}, i \partial_{r}\right) \tilde{\chi} d r d \theta d \phi
$$


where $\operatorname{diag}\left(i \partial_{r},-i \partial_{r},-i \partial_{r}, i \partial_{r}\right)$ stays for the diagonal matrix whose non vanishing entries are explicitly given. The $\theta$ derivatives come out from $\mathcal{L}_{ \pm}$and appear in the form

where

$$
\int \sin \theta \frac{1}{\sqrt{\Delta_{r}}}{ }^{t} \tilde{\psi}^{*} E \frac{1}{\sqrt{\sin \theta}} \partial_{\theta}(\sqrt{\sin \theta} \tilde{\chi}) d r d \theta d \phi
$$

$$
E=\left(\begin{array}{cccc}
0 & -1 & 0 & 0 \\
1 & 0 & 0 & 0 \\
0 & 0 & 0 & 1 \\
0 & 0 & -1 & 0
\end{array}\right)
$$

From these expressions the symmetry of the operator $\hat{H}_{0}$ is easily checked.

We prove that there exists an unitary isomorphism between $\mathcal{H}_{<>}$and $\mathcal{H}_{()}$. We follow a line of thought which is strictly analogous to the one allowing to prove the unitary equivalence between the Hilbert space $L^{2}(a, b, q)$ and $L^{2}(a, b)$, where the former space has measure $q(x) d x$ and $q:(a, b) \rightarrow \mathbb{R}$ is a measurable, almost everywhere positive and locally integrable function. Cf. [18], pp. 247-248.

Lemma 1. The map $V_{\Omega}: \mathcal{H}_{<>} \mapsto \mathcal{H}_{()}$defined by

$$
\left(V_{\Omega} \psi\right)(r, \theta, \phi)=\Omega(r, \theta) \psi(r, \theta, \phi)
$$

is an unitary isomorphism of Hilbert spaces.

Proof. One has

$$
\left(V_{\Omega} \psi \mid V_{\Omega} \chi\right)=(\hat{\Omega} \psi \mid \hat{\Omega} \chi)=\left(\psi \mid \hat{\Omega}^{2} \chi\right)=\langle\psi \mid \chi\rangle .
$$

Then, due to the aforementioned properties of $\hat{\Omega}$ and $\hat{\Omega}^{-1}, V_{\Omega}$ is an (unitary) isomorphism of Hilbert spaces [18. Note also that $V_{\Omega}^{*}: \mathcal{H}_{()} \mapsto \mathcal{H}_{<>}$and that $V_{\Omega}^{*}$ acts as a multiplication operator by $\Omega^{-1}(r, \theta)$.

It is useful to introduce $V_{\Omega} \hat{H} V_{\Omega}^{-1}$ which is defined on the domain $V_{\Omega} \mathcal{D} \subset \mathcal{H}_{()}$and which is unitarily equivalent to the operator $\hat{H}$ defined on $\mathcal{D} \subset \mathcal{H}_{<>}$. Then the problem of the essential selfadjointness of $\hat{H}$ in $\mathcal{D} \subset \mathcal{H}<>$ is equivalent to the problem of essential selfadjointness of $V_{\Omega} \hat{H} V_{\Omega}^{-1}$ on $V_{\Omega} \mathcal{D} \subset \mathcal{H}_{()}$. By taking into account $\hat{H}=\hat{\Omega}^{-2} \hat{H}_{0}$ the aforementioned problem amounts to the essential selfadjointness of $\hat{\Omega}^{-1} \hat{H}_{0} \hat{\Omega}^{-1}$ in $V_{\Omega} \mathcal{D} \subset \mathcal{H}()$.

Now we can prove the following result.

Theorem 1. $\hat{H}$ is essentially selfadjoint if and only if $\hat{H}_{0}$ is essentially selfadjoint.

Proof. The following results are useful:

a) let $\hat{A}$ be a densely defined operator from $\mathcal{H}_{1}$ to $\mathcal{H}_{2}$ and let $\hat{B}$ be a bounded operator from $\mathcal{H}_{2}$ to $\mathcal{H}_{3}$. Then $(\hat{B} \hat{A})^{*}=\hat{A}^{*} \hat{B}^{*}$.

b) Let $\hat{C}, \hat{D}$ be densely defined operators defined from $\mathcal{H}_{1}$ to $\mathcal{H}_{2}$ and from $\mathcal{H}_{2}$ to $\mathcal{H}_{3}$ respectively. Assume that $\hat{D} \hat{C}$ is densely defined from $\mathcal{H}_{1}$ to $\mathcal{H}_{3}$, and assume that $\hat{C}$ is injective with $\hat{C}^{-1} \in \mathcal{B}\left(\mathcal{H}_{2}, \mathcal{H}_{1}\right)$. Then $(\hat{D} \hat{C})^{*}=\hat{C}^{*} \hat{D}^{*}$ (cf. ex. 4.18 , p.74 of [18]).

In our case, in order to apply (a) one identifies $\hat{B}$ with $\hat{\Omega}^{-1}$ and $\hat{A}$ with the product $\hat{H}_{0} \hat{\Omega}^{-1}$; moreover, in order to apply (b) one identifies $\hat{C}$ with $\hat{\Omega}^{-1}$ and $\hat{D}$ with $\hat{H}_{0}$. As a consequence, one finds that the essential selfadjointness condition, which amounts to $\left(\hat{\Omega}^{-1} \hat{H}_{0} \hat{\Omega}^{-1}\right)^{*}=\left(\hat{\Omega}^{-1} \hat{H}_{0} \hat{\Omega}^{-1}\right)^{* *}$, is implemented if and only if $\hat{H}_{0}^{*}=\hat{H}_{0}^{* *}$, i.e. if and only if $\hat{H}_{0}$ is essentially selfadjoint on $\mathcal{D} \subset \mathcal{H}_{()}$. (Indeed, from (a) we know that $\left(\hat{\Omega}^{-1} \hat{H}_{0} \hat{\Omega}^{-1}\right)^{*}=\left(\hat{H}_{0} \hat{\Omega}^{-1}\right)^{*} \hat{\Omega}^{-1}$, and from (b) one finds $\left(\hat{H}_{0} \hat{\Omega}^{-1}\right)^{*} \hat{\Omega}^{-1}=\hat{\Omega}^{-1} \hat{H}_{0}^{*} \hat{\Omega}^{-1}$. Analogously, $\left.\left(\hat{\Omega}^{-1} \hat{H}_{0} \hat{\Omega}^{-1}\right)^{* *}=\left[\left(\hat{\Omega}^{-1} \hat{H}_{0} \hat{\Omega}^{-1}\right)^{*}\right]^{*}=\left[\hat{\Omega}^{-1} \hat{H}_{0}^{*} \hat{\Omega}^{-1}\right]^{*}=\left(\hat{H}_{0}^{*} \hat{\Omega}^{-1}\right)^{*} \hat{\Omega}^{-1}=\hat{\Omega}^{-1} \hat{H}_{0}^{* *} \hat{\Omega}^{-1}.\right)$

To sum up, we have shown that the essential selfadjointness of $\hat{H}$ on $\mathcal{D} \subset \mathcal{H}_{<>}$is equivalent to the essential selfadjointness of $\hat{H}_{0}$ on $\mathcal{D} \subset \mathcal{H}_{()}$. Furthermore, if $\hat{T}_{H_{0}}$ is a selfadjoint realization of $H_{0}$, to be defined on $D\left(\hat{T}_{H_{0}}\right):=\mathfrak{D} \subset \mathcal{H}_{()}$, we introduce $\hat{T}_{H}:=\hat{\Omega}^{-2} \hat{T}_{H_{0}}$ on $\mathcal{H}_{<>}$. One can show analogously that $\hat{T}_{H}$ is a selfadjoint realization of $H$ on $\mathfrak{D} \subset \mathcal{H}_{<>}$. 
(Proof: by the above unitary isomorphism (cf. Lemma 1) let us define $\tilde{\hat{T}}_{H}=\hat{\Omega} \hat{T}_{H} \hat{\Omega}^{-1}=\hat{\Omega}^{-1} \hat{T}_{H_{0}} \hat{\Omega}^{-1}$ on $\Omega \mathfrak{D}$. One finds easily (cf. Theorem 1) $\tilde{\hat{T}}_{H}^{*}=\hat{\Omega}^{-1} \hat{T}_{H_{0}}^{*} \hat{\Omega}^{-1}=\hat{\Omega}^{-1} \hat{T}_{H_{0}} \hat{\Omega}^{-1}=\tilde{\hat{T}}_{H}$, and then $\tilde{\hat{T}}_{H}$ is selfadjoint. As a consequence $\hat{T}_{H}=\hat{\Omega}^{-2} \hat{T}_{H_{0}}$ on $\mathfrak{D} \subset \mathcal{H}_{<>}$is selfadjoint too, and $\left.\hat{\Omega}^{-2} \hat{T}_{H_{0}} f=H f, f \in \mathfrak{D}\right)$.

5.1. Essential selfadjointness of $\hat{H}_{0}$ on $\mathcal{D}=C_{0}^{\infty}\left(\left(r_{+}, \infty\right) \times S^{2}\right)^{4} \subset \mathcal{H}_{()}$. It is useful to recall that $\mathcal{H}_{()} \simeq L^{2}\left(\left(r_{+}, \infty\right), \frac{r^{2}+a^{2}}{\Delta_{r}} d r\right)^{2} \otimes L^{2}\left((0, \pi), \frac{\sin (\theta)}{\sqrt{\Delta_{\theta}}} d \theta\right)^{2} \otimes L^{2}(0,2 \pi)$, where the scalar product is the "usual" one $\left((f, g):=\int d \mu f^{*} g\right.$; for short, the measure is indicated by $d \mu$ and $f, g$ are scalar or vector functions depending on the case). We introduce the following unitary operator (cf. [19]) $V: \mathcal{H}_{()} \rightarrow \mathcal{H}_{()}$:

$$
V=\frac{1}{\sqrt{2}}\left(\begin{array}{cccc}
0 & -i & 0 & i \\
i & 0 & -i & 0 \\
0 & -1 & 0 & -1 \\
-1 & 0 & -1 & 0
\end{array}\right)
$$

and then we consider the operator $V \hat{H}_{0} V^{*}$ on $V \mathcal{D}$. The latter operator is particularly suitable for the study of essential selfadjointness by means of variable separation. One finds:

$$
V \tilde{\mathcal{R}} V^{*}=\left(\begin{array}{cccc}
\frac{1}{2}\left(\mathcal{E}_{-}-\mathcal{E}_{+}\right)+\mu(r) & 0 & -\frac{1}{2} i\left(\mathcal{E}_{+}+\mathcal{E}_{-}\right) & 0 \\
0 & \frac{1}{2}\left(\mathcal{E}_{-}-\mathcal{E}_{+}\right)+\mu(r) & 0 & -\frac{1}{2} i\left(\mathcal{E}_{+}+\mathcal{E}_{-}\right) \\
\frac{1}{2} i\left(\mathcal{E}_{+}+\mathcal{E}_{-}\right) & 0 & \frac{1}{2}\left(\mathcal{E}_{-}-\mathcal{E}_{+}\right)-\mu(r) & 0 \\
0 & \frac{1}{2} i\left(\mathcal{E}_{+}+\mathcal{E}_{-}\right) & 0 & \frac{1}{2}\left(\mathcal{E}_{-}-\mathcal{E}_{+}\right)-\mu(r)
\end{array}\right)
$$

where $\mu(r):=\mu \frac{r \sqrt{\Delta_{r}}}{r^{2}+a^{2}}$; moreover, it holds

$$
V \tilde{\mathcal{A}} V^{*}=\left(\begin{array}{cccc}
0 & 0 & -\mu a \cos (\theta) \frac{\sqrt{\Delta_{r}}}{r^{2}+a^{2}} & i \mathcal{M}_{+} \\
0 & 0 & i \mathcal{M}_{-} & \mu a \cos (\theta) \frac{\sqrt{\Delta_{r}}}{r^{2}+a^{2}} \\
-\mu a \cos (\theta) \frac{\sqrt{\Delta_{r}}}{r^{2}+a^{2}} & i \mathcal{M}_{+} & 0 & 0 \\
i \mathcal{M}_{-} & \mu a \cos (\theta) \frac{\sqrt{\Delta_{r}}}{r^{2}+a^{2}} & 0 & 0
\end{array}\right) .
$$

Then, from the explicit expressions of $\mathcal{E}_{ \pm}$and of $\mathcal{M}_{ \pm}$one obtains

$$
V \tilde{\mathcal{R}} V^{*}=\left(\begin{array}{cc}
\frac{1}{r^{2}+a^{2}}\left(i a \Xi \partial_{\phi}+e q_{e} r+\mu r \sqrt{\Delta_{r}}\right) \mathbb{I} & \frac{\Delta_{r}}{r^{2}+a^{2}} \partial_{r} \mathbb{I} \\
-\frac{\Delta_{r}}{r^{2}+a^{2}} \partial_{r} \mathbb{I} & \frac{1}{r^{2}+a^{2}}\left(i a \Xi \partial_{\phi}+e q_{e} r-\mu r \sqrt{\Delta_{r}}\right) \mathbb{I}
\end{array}\right)
$$

and

$$
V \tilde{\mathcal{A}} V^{*}=\frac{\sqrt{\Delta_{r}}}{r^{2}+a^{2}}\left(\begin{array}{ll}
\mathbb{O} & \mathbb{U} \\
\mathbb{U} & \mathbb{O}
\end{array}\right)
$$

where $\mathbb{U}$ is the $2 \times 2$ matrix formal differential expression

$$
\mathbb{U}=\left(\begin{array}{cc}
-\mu a \cos (\theta) & i \sqrt{\Delta_{\theta}}\left(\partial_{\theta}+\frac{1}{2} \cot (\theta)+g\right) \\
\left.i \sqrt{\Delta_{\theta}}\left(\partial_{\theta}+\frac{1}{2} \cot (\theta)-g\right)\right) & \mu a \cos (\theta)
\end{array}\right),
$$

with $g:=i \frac{1}{\Delta_{\theta} \sin (\theta)} \Xi \partial_{\phi}-\frac{1}{\Delta_{\theta}} q_{m} e \cot (\theta)$. We define also $\hat{\mathbb{U}}$ to be a differential operator in the Hilbert space $L^{2}\left((0, \pi), \frac{\sin (\theta)}{\sqrt{\Delta_{\theta}}} d \theta\right)^{2} \otimes L^{2}(0,2 \pi)$, with domain $D(\hat{\mathbb{U}})=L\left\{C_{0}^{\infty}(0, \pi)^{2} \times C_{0}^{\infty}(0,2 \pi)\right\} \quad(L\{\cdot\}$ stays for the linear hull), and $\hat{\mathbb{U}} S=\mathbb{U} S$ for $S \in D(\hat{\mathbb{U}})$.

As a consequence of the above manipulations, we obtain $V \hat{H}_{0} V^{*}$ on $V \mathcal{D}$, with $V \hat{H}_{0} V^{*} \chi=V H_{0} V^{*} \chi, \chi \in \mathcal{D}$, where the formal differential expression $V H_{0} V^{*}$ is:

$$
V H_{0} V^{*}=\left(\begin{array}{cc}
\frac{1}{r^{2}+a^{2}}\left(i a \Xi \partial_{\phi}+e q_{e} r+\mu r \sqrt{\Delta_{r}}\right) \mathbb{I} & \frac{\Delta_{r}}{r^{2}+a^{2}} \partial_{r} \mathbb{I}+\frac{\sqrt{\Delta_{r}}}{r^{2}+a^{2}} \mathbb{U} \\
-\frac{\Delta_{r}}{r^{2}+a^{2}} \partial_{r} \mathbb{I}+\frac{\sqrt{\Delta_{r}}}{r^{2}+a^{2}} \mathbb{U} & \frac{1}{r^{2}+a^{2}}\left(i a \Xi \partial_{\phi}+e q_{e} r-\mu r \sqrt{\Delta_{r}}\right) \mathbb{I}
\end{array}\right) .
$$


We consider the subset $\tilde{\mathcal{D}}$ of $\mathcal{D}$ which contains finite linear combinations of functions of the following form:

$$
\chi(r, \theta, \phi)=\varepsilon(\phi)\left(\begin{array}{c}
R_{1}(r) S_{2}(\theta) \\
R_{2}(r) S_{1}(\theta) \\
R_{2}(r) S_{2}(\theta) \\
R_{1}(r) S_{1}(\theta)
\end{array}\right),
$$

where $\varepsilon(\phi) \in C_{0}^{\infty}(0,2 \pi), R(r):=\left(\begin{array}{c}R_{1}(r) \\ R_{2}(r)\end{array}\right) \in C_{0}^{\infty}\left(r_{+}, \infty\right)^{2}$ and $S(\theta):=\left(\begin{array}{c}S_{1}(\theta) \\ S_{2}(\theta)\end{array}\right) \in C_{0}^{\infty}(0, \pi)^{2}$. Then one obtains

$$
V \chi(r, \theta, \phi)=\varepsilon(\phi) \frac{1}{\sqrt{2}}\left(\begin{array}{c}
-i\left(R_{2}(r)-R_{1}(r)\right) S_{1}(\theta) \\
-i\left(R_{2}(r)-R_{1}(r)\right) S_{2}(\theta) \\
-\left(R_{2}(r)+R_{1}(r)\right) S_{1}(\theta) \\
-\left(R_{2}(r)+R_{1}(r)\right) S_{2}(\theta)
\end{array}\right)
$$

The subspace $L_{k}$ spanned by the eigenfunctions $e^{-i k \phi}, k \in \mathbb{Z}+\frac{1}{2}$ of the the selfadjoint operator $i \partial_{\phi}$ with antiperiodic boundary conditions at 0 and at $2 \pi$ is such that $L^{2}\left(\left(r_{+}, \infty\right), \frac{r^{2}+a^{2}}{\Delta_{r}} d r\right)^{2} \otimes L^{2}\left((0, \pi), \frac{\sin (\theta)}{\sqrt{\Delta_{\theta}}} d \theta\right)^{2} \otimes L_{k}$ is a reducing subspace for $V \hat{H}_{0} V^{*}$. Moreover $i \partial_{\phi}$ and $\hat{\mathbb{U}}$ trivially commute. Let $\hat{\mathbb{U}}_{k} \otimes I_{k}$ be the operator obtained by restricting $\hat{\mathbb{U}}$ to $C_{0}^{\infty}(0, \pi)^{2} \otimes L_{k}\left(I_{k}\right.$ is the identity operator on $\left.L_{k}\right)$; one finds that $\hat{\mathbb{U}}_{k}$, whose formal differential expression is

$$
\mathbb{U}_{k}=\left(\begin{array}{cc}
-\mu a \cos (\theta) & i \sqrt{\Delta_{\theta}}\left(\partial_{\theta}+\frac{1}{2} \cot (\theta)+b_{k}(\theta)\right) \\
\left.i \sqrt{\Delta_{\theta}}\left(\partial_{\theta}+\frac{1}{2} \cot (\theta)-b_{k}(\theta)\right)\right) & \mu a \cos (\theta)
\end{array}\right),
$$

where $b_{k}(\theta):=\frac{1}{\Delta_{\theta} \sin (\theta)} \Xi k-\frac{1}{\Delta_{\theta}} q_{m} e \cot (\theta)$, is essentially selfadjoint on $C_{0}^{\infty}(0, \pi)^{2}$ for any $k \in \mathbb{Z}+\frac{1}{2}$ for $\frac{q_{m} e}{\Xi} \in \mathbb{Z}$ (see sect. [5.1.1] for details). Note also that $\hat{\mathbb{U}}=\oplus_{k} \hat{\mathbb{U}}_{k} \otimes I_{k}$. If one considers the selfadjoint extension $\overline{\mathbb{U}}_{k}$ of $\hat{\mathbb{U}}_{k}$, one can show that $\overline{\hat{U}}_{k}$ has purely discrete spectrum which is simple (see section 6.1 and see also Appendix B).

Let us introduce the (normalized) eigenfunctions $S_{k ; j}(\theta):=\left(\begin{array}{c}S_{1 k ; j}(\theta) \\ S_{2 k ; j}(\theta)\end{array}\right)$ of the operator $\overline{\hat{\mathbb{U}}}_{k}$ :

$$
\overline{\hat{U}}_{k}\left(\begin{array}{c}
S_{1 k ; j}(\theta) \\
S_{2 k ; j}(\theta)
\end{array}\right)=\lambda_{k ; j}\left(\begin{array}{c}
S_{1 k ; j}(\theta) \\
S_{2 k ; j}(\theta)
\end{array}\right)
$$

then $\mathcal{H}_{k, j}:=L^{2}\left(\left(r_{+}, \infty\right), \frac{r^{2}+a^{2}}{\Delta_{r}} d r\right)^{2} \otimes M_{k, j}$, where $M_{k, j}:=\left\{F_{k ; j}(\theta, \phi)\right\}$, with $F_{k ; j}(\theta, \phi):=S_{k ; j}(\theta) \frac{e^{-i k \phi}}{\sqrt{2 \pi}}$, is a reducing subspace for $V \hat{H}_{0} V^{*}$. Let us define $D_{k, j}:=\tilde{\mathcal{D}} \cap \mathcal{H}_{k, j}$. Then $\left.V \hat{H}_{0} V^{*}\right|_{D_{k, j}}$ is such that $V \hat{H}_{0} V^{*}(V \chi)=$ $\omega(V \chi)$ becomes

$$
\left(\begin{array}{cc}
\frac{1}{r^{2}+a^{2}}\left(a \Xi k+e q_{e} r+\mu r \sqrt{\Delta_{r}}\right) \mathbb{I} & \frac{\Delta_{r}}{r^{2}+a^{2}} \partial_{r} \mathbb{I}+\frac{\sqrt{\Delta_{r}}}{r^{2}+a^{2}} \lambda_{k ; j} \mathbb{I} \\
-\frac{\Delta_{r}}{r^{2}+a^{2}} \partial_{r} \mathbb{I}+\frac{\sqrt{\Delta_{r}}}{r^{2}+a^{2}} \lambda_{k ; j} \mathbb{I} & \frac{1}{r^{2}+a^{2}}\left(a \Xi k+e q_{e} r-\mu r \sqrt{\Delta_{r}}\right) \mathbb{I}
\end{array}\right) V \chi=\omega V \chi,
$$

which is equivalent to the following $2 \times 2$ Dirac system for the radial part:

$$
\left(\begin{array}{cc}
\frac{1}{r^{2}+a^{2}}\left(a \Xi k+e q_{e} r+\mu r \sqrt{\Delta_{r}}\right) & \frac{\Delta_{r}}{r^{2}+a^{2}} \partial_{r}+\frac{\sqrt{\Delta_{r}}}{r^{2}+a^{2}} \lambda_{k ; j} \\
-\frac{\Delta_{r}}{r^{2}+a^{2}} \partial_{r}+\frac{\sqrt{\Delta_{r}}}{r^{2}+a^{2}} \lambda_{k ; j} & \frac{1}{r^{2}+a^{2}}\left(a \Xi k+e q_{e} r-\mu r \sqrt{\Delta_{r}}\right)
\end{array}\right)\left(\begin{array}{c}
X_{1}(r) \\
X_{2}(r)
\end{array}\right)=\omega\left(\begin{array}{c}
X_{1}(r) \\
X_{2}(r)
\end{array}\right),
$$

where

$$
\left(\begin{array}{c}
X_{1}(r) \\
X_{2}(r)
\end{array}\right):=\frac{1}{\sqrt{2}}\left(\begin{array}{c}
-i\left(R_{2}(r)-R_{1}(r)\right) \\
-\left(R_{2}(r)+R_{1}(r)\right)
\end{array}\right) .
$$

Then we obtain $\mathcal{H}_{()}=\oplus_{k, j} \mathcal{H}_{k, j}$, and we also obtain the following orthogonal decomposition [20] (also called partial wave decomposition) of the operator $V \hat{H}_{0} V^{*}$ :

$$
V \hat{H}_{0} V^{*}=\bigoplus_{k, j} \hat{h}_{k, j} \otimes I_{k, j}
$$


where $I_{k, j}$ stays for the identity operator on $M_{k, j}$ and $\hat{h}_{k, j}$, which is defined on $\mathcal{D}_{k, j}:=C_{0}^{\infty}\left(r_{+}, \infty\right)^{2}$, has the following formal expression:

$$
h_{k, j}:=\left(\begin{array}{cc}
\frac{1}{r^{2}+a^{2}}\left(a \Xi k+e q_{e} r+\mu r \sqrt{\Delta_{r}}\right) & \frac{\Delta_{r}}{r^{2}+a^{2}} \partial_{r}+\frac{\sqrt{\Delta_{r}}}{r^{2}+a^{2}} \lambda_{k ; j} \\
-\frac{\Delta_{r}}{r^{2}+a^{2}} \partial_{r}+\frac{\sqrt{\Delta_{r}}}{r^{2}+a^{2}} \lambda_{k ; j} & \frac{1}{r^{2}+a^{2}}\left(a \Xi k+e q_{e} r-\mu r \sqrt{\Delta_{r}}\right)
\end{array}\right)
$$

In the following subsections, we study essential selfadjointness conditions both for the angular momentum operator $\mathbb{U}$ and for the reduced Hamiltonian $\hat{h}_{k, j}$. Note that if $\hat{h}_{k, j}$ is essentially selfadjoint on $\mathcal{D}_{k, j}$, then $V \hat{H}_{0} V^{*}$ is essentially selfadjoint on the linear hull $L\left\{\mathcal{D}_{k, j} \otimes M_{k, j} ; k, j\right\}[18]$.

5.1.1. Essential selfadjointness of $\hat{\mathbb{U}}_{k}$. The operator $\mathbb{U}_{k}$ is formally selfadjoint in $L^{2}\left((0, \pi), \frac{\sin (\theta)}{\sqrt{\Delta_{\theta}}} d \theta\right)^{2}$ whose elements are indicated by $S(\theta):=\left(\begin{array}{c}S_{1}(\theta) \\ S_{2}(\theta)\end{array}\right)$. The conditions for the essential selfadjointness of $\hat{\mathbb{U}}_{k}$ on $C_{0}^{\infty}(0, \pi)^{2}$ are determined in the following.

By means of the unitary transformation

$$
W:=\left(\begin{array}{cc}
0 & -i \\
1 & 0
\end{array}\right)
$$

the operator $\hat{\mathbb{U}}_{k}$ has a formal differential expression which is carried into the following form which corresponds to a Dirac system [21]:

$$
W \mathbb{U}_{k} W^{*}=\left(\begin{array}{cc}
\mu a \cos (\theta) & \sqrt{\Delta_{\theta}}\left(\partial_{\theta}+\frac{1}{2} \cot (\theta)-b_{k}(\theta)\right) \\
\sqrt{\Delta_{\theta}}\left(-\partial_{\theta}-\frac{1}{2} \cot (\theta)-b_{k}(\theta)\right) & -\mu a \cos (\theta)
\end{array}\right) .
$$

A further Liouville unitary transformation $R: L^{2}\left((0, \pi), \frac{\sin (\theta)}{\sqrt{\Delta_{\theta}}} d \theta\right)^{2} \rightarrow L^{2}\left((0, \pi), \frac{1}{\sqrt{\Delta_{\theta}}} d \theta\right)^{2}$,

$$
(R S)(\theta):=(\sin (\theta))^{\frac{1}{2}} S(\theta)=: \Theta(\theta)
$$

(cf. 19] for the Kerr-Newman case) allows us to determine for $\lambda \in \mathbb{C}$ if the limit point case or the limit circle case is implemented according to Weyl's alternative [21] by studying the differential system $R W \mathbb{U}_{k} W^{*} R^{*} \Theta=\lambda \Theta$, i.e.

$$
\left(\begin{array}{cc}
\mu a \cos (\theta) & \sqrt{\Delta_{\theta}}\left(\partial_{\theta}-\frac{\Xi k}{\Delta_{\theta} \sin (\theta)}+\frac{1}{\Delta_{\theta}} q_{m} e \cot (\theta)\right) \\
\sqrt{\Delta_{\theta}}\left(-\partial_{\theta}-\frac{\Xi k}{\Delta_{\theta} \sin (\theta)}+\frac{1}{\Delta_{\theta}} q_{m} e \cot (\theta)\right) & -\mu a \cos (\theta)
\end{array}\right) \Theta=\lambda \Theta .
$$

We shall determine for $\lambda \in \mathbb{C}$ if the limit point case or the limit circle case is implemented according to Weyl's alternative [21]. The above equation amounts to a first order differential system which displays a first kind singularity both at $\theta=0$ and at $\theta=\pi[22,23$. In the former case, one can write

$$
\theta \partial_{\theta} \Theta=N \Theta
$$

where the smooth matrix $N$ is regular as $\theta \rightarrow 0^{+}$and

$$
N_{0}:=\lim _{\theta \rightarrow 0^{+}} N=\left(\begin{array}{cc}
-k+\frac{q_{m} e}{\Xi} & 0 \\
0 & k-\frac{q_{m} e}{\Xi}
\end{array}\right) .
$$

Then the eigenvalues of $N_{0}$ are $\pm \nu$ with $\nu=k-\frac{q_{m} e}{\Xi}$. One can find two linearly independent solutions $\Theta_{1}, \Theta_{2}$ near $\theta=0$ such that

$$
\Theta_{1}=\theta^{\nu} h_{1}(\theta)
$$

and

$$
\Theta_{2}=\theta^{-\nu}\left(h_{2}(\theta)+\log (\theta) h_{3}(\theta)\right)
$$

where $h_{i}(\theta):=\left(\begin{array}{c}h_{1 ; i}(\theta) \\ h_{2 ; i}(\theta)\end{array}\right)$ are analytic near $\theta=0$ for $i=1,2,3$ and $h_{3} \neq 0$ only for $2 \frac{q_{m} e}{\Xi}$ integer [23]. We recall that $k=n+\frac{1}{2}$. It is easy to conclude that the limit point case [21] occurs at $\theta=0$ only for

$$
n \leq \frac{q_{m} e}{\Xi}-1, \quad \text { and } \quad n \geq \frac{q_{m} e}{\Xi} .
$$


The study at $\theta=\pi$ is analogous. Let us define $\alpha=\pi-\theta$. Then it is straightforward to show that also for $\alpha=0$ there is a first kind singularity by studying

$$
\alpha \partial_{\alpha} \Theta=M \Theta,
$$

where the smooth matrix $M$ is regular as $\alpha \rightarrow 0^{+}$and

$$
M_{0}:=\lim _{\alpha \rightarrow 0^{+}} M=\left(\begin{array}{cc}
k+\frac{q_{m} e}{\Xi} & 0 \\
0 & -k-\frac{q_{m} e}{\Xi}
\end{array}\right) .
$$

Then the eigenvalues of $M_{0}$ are $\pm \rho_{0}$, with $\rho_{0}=k+\frac{q_{m} e}{\Xi}$. One can find two linearly independent solutions near $\alpha=0$ as above. Then the limit point case occurs at $\theta=\pi$ only for

$$
n \geq-\frac{q_{m} e}{\Xi}, \quad \text { and } \quad n \leq-\frac{q_{m} e}{\Xi}-1 .
$$

From (5.34) and (5.37) we see that if $\frac{q_{m} e}{\Xi} \in \mathbb{Z}$ then the essential selfadjointness in $C_{0}^{\infty}(0, \pi)^{2}$ is obtained for any $n \in \mathbb{Z}$. See also Appendix $₫$ for a further discussion.

Note that if $\frac{q_{m} e}{\Xi} \notin \mathbb{Z}$ then the essential selfadjointness of $\mathbb{U}_{k}$ is obtained for

$$
n \in \mathbb{Z}-\left\{\left[-1-\left|\frac{q_{m} e}{\Xi}\right|\right],\left[\left|\frac{q_{m} e}{\Xi}\right|\right]\right\}
$$

where with $[z]$ we mean the integer part of $z$. Then there would be some $k=n+\frac{1}{2}$, with $n \in\left\{\left[-1-\left|\frac{q_{m} e}{\Xi}\right|\right]\right.$, $\left.\left[\left|\frac{q_{m} e}{\Xi}\right|\right]\right\}$, such that essential selfadjointness does not occur on $C_{0}^{\infty}(0, \pi)^{2}$. We limit ourselves to impose herein for the product $\frac{q_{m} e}{\Xi}$ to be integer: this has a nice interpretation, because it can be related to the Dirac quantization condition (we recall that $\frac{q_{m}}{\Xi}=Q_{m}$ is the magnetic charge of the black hole); see also Appendix $\mathrm{A}$.

As a consequence, we have shown that the following result holds:

Theorem 2. $\hat{\mathbb{U}}_{k}$ is essentially self adjoint on $C_{0}^{\infty}(0, \pi)^{2}$ for any $k=n+\frac{1}{2}, n \in \mathbb{Z}$ iff $\frac{q_{m} e}{\Xi} \in \mathbb{Z}$.

Note that, for $q_{m}=0$ one recovers the same condition as for the standard Kerr-Newman case discussed in Refs. [19, 12.

5.1.2. Essential selfadjointness of $\hat{h}_{k, j}$. The differential expression $h_{k, j}$ is formally selfadjoint in the Hilber space $L^{2}\left(\left(r_{+}, \infty\right), \frac{r^{2}+a^{2}}{\Delta_{r}} d r\right)^{2}$. In order to study the essential selfadjointness of the reduced Hamiltonian in $C_{0}^{\infty}\left(r_{+}, \infty\right)^{2}$ one has to check if the limit point case occurs both at the event horizon $r=r_{+}$and at $r=\infty$. In the former case, it is useful introducing the following reparameterization of the metric in the non-extremal case:

$$
\Delta_{r}=\frac{1}{l^{2}}\left(r-r_{+}\right)\left(r-r_{-}\right)\left(r^{2}+\left(r_{+}+r_{-}\right) r+r_{+}^{2}+r_{-}^{2}+r_{+} r_{-}+a^{2}+l^{2}\right),
$$

where the parameters $m, z^{2}, a, l$ are replaced by $r_{+}, r_{-}, a, l$. One easily finds:

$$
\begin{aligned}
m & =\frac{1}{2 l^{2}}\left(r_{+}+r_{-}\right)\left(r_{+}^{2}+r_{-}^{2}+a^{2}+l^{2}\right) \\
z^{2} & =\frac{1}{2 l^{2}} r_{+} r_{-}\left(r_{+}^{2}+r_{-}^{2}+r_{+} r_{-}+a^{2}+l^{2}\right)-a^{2} .
\end{aligned}
$$

This is a good reparameterization, indeed the Jacobian $J$ of the transformation is

$$
J=\frac{1}{2 l^{4}}\left(3 r_{+}^{2}+r_{-}^{2}+2 r_{+} r_{-}+a^{2}+l^{2}\right)\left(r_{+}^{2}+3 r_{-}^{2}+2 r_{+} r_{-}+a^{2}+l^{2}\right)\left(r_{+}-r_{-}\right)
$$

which is strictly positive for non extremal black holes.

It is also evident that in the extremal case, where $r_{-}=r_{+}$a reparameterization analogous to (5.39) is available:

$$
\Delta_{r}^{\text {extr }}=\frac{1}{l^{2}}\left(r-r_{+}\right)^{2}\left(r^{2}+2 r_{+} r+3 r_{+}^{2}+a^{2}+l^{2}\right),
$$

by taking into account that in the extremal case the parameters $m, z^{2}, a, l$ are no more independent (cf. e.g. $(2.20)$ ).

We show that the following result holds: 
Theorem 3. $\hat{h}_{k, j}$ is essentially selfadjoint on $C_{0}^{\infty}\left(r_{+}, \infty\right)^{2}$ iff $\mu l \geq \frac{1}{2}$.

Proof. We choose the tortoise coordinate $y$ defined by

$$
d y=-\frac{r^{2}+a^{2}}{\Delta_{r}} d r
$$

and choose a free integration constant in such a way that $y \in(0, \infty)$. It holds $y \rightarrow \infty \Leftrightarrow r \rightarrow r_{+}{ }^{+}$. Then we get

$$
h_{k, j}=\left(\begin{array}{cc}
0 & -\partial_{y} \\
\partial_{y} & 0
\end{array}\right)+V(r(y))
$$

and the corollary to thm. 6.8 p.99 in [21] ensures that the limit point case holds for $h_{k, j}$ at $y=\infty$.

It is also useful to point out that it holds

$$
\lim _{y \rightarrow \infty} V(r(y))=\left(\begin{array}{cc}
\frac{1}{r_{+}^{2}+a^{2}}\left(a k \Xi+e q_{e} r_{+}\right) & 0 \\
0 & \frac{1}{r_{+}^{2}+a^{2}}\left(a k \Xi+e q_{e} r_{+}\right)
\end{array}\right):=\left(\begin{array}{cc}
\varphi_{+} & 0 \\
0 & \varphi_{+}
\end{array}\right) .
$$

The only problem can be found at $r=\infty$. The differential equation $h_{k, j} X=\omega X$ amounts to the following differential system:

$$
\partial_{r} X=\left(\begin{array}{cc}
\frac{\lambda_{k ; j}}{\sqrt{\Delta_{r}}} & -\frac{\omega\left(r^{2}+a^{2}\right)}{\Delta_{r}}-\frac{\mu r}{\sqrt{\Delta_{r}}}+\frac{P(r)}{\Delta_{r}} \\
\frac{\omega\left(r^{2}+a^{2}\right)}{\Delta_{r}}-\frac{\mu r}{\sqrt{\Delta_{r}}}-\frac{P(r)}{\Delta_{r}} & -\frac{\lambda_{k ; j}}{\sqrt{\Delta_{r}}}
\end{array}\right) X
$$

where $X(r):=\left(\begin{array}{c}X_{1}(r) \\ X_{2}(r)\end{array}\right)$ and $P(r)=a k \Xi+e q_{e} r$. In order to study the behavior of this differential system at $r=\infty$ it is useful to introduce momentarily $x=\frac{1}{r}$. Then one obtains

$$
x \partial_{x} X=G(x) X,
$$

where the smooth matrix $G(x)$ is regular as $x \rightarrow 0^{+}$and

$$
\lim _{x \rightarrow 0^{+}} G(x)=\left(\begin{array}{cc}
0 & \mu l \\
\mu l & 0
\end{array}\right) .
$$

A singularity of the first kind is found, with eigenvalues $w_{ \pm}= \pm \mu l$. As in the previous subsection, we can conclude that the limit point case occurs at $r=\infty$ iff

$$
\int_{c}^{\infty} \frac{d r}{r^{2}} r^{ \pm 2 \mu l}=\infty
$$

For $\mu>0$ as in the physical interesting case, the limit point case occurs for $\mu l \geq \frac{1}{2}$, which is also the required essential selfadjointness condition for the reduced Hamiltonian.

If $\mu l<\frac{1}{2}$, there is a 1-parameter family of selfadjoint extensions $\hat{t}_{k, j}$ of $\hat{h}_{k, j}[21$.

\section{The eigenvalue equation.}

We limit our considerations to the case $\mu l \geq \frac{1}{2}$, for which we know that $\hat{H}$ is essentially selfadjoint on $\mathcal{D}=C_{0}^{\infty}\left(\left(r_{+}, \infty\right) \times S^{2}\right)^{4} \subset \mathcal{H}_{<>}$, and then there exists a unique selfadjoint extension $\overline{\hat{H}}$ on $\mathfrak{D}$. See also the conclusions. The following relation holds between the eigenvalue equation for $\overline{\hat{H}}$ and the differential system one finds by separating the variables as in the Chandrasekhar-like trick. We have that $\overline{\hat{H}}=\hat{\Omega}^{-2} \overline{\hat{H}}_{0}$. From

$$
\overline{\hat{H}} \psi=\omega \psi
$$

one obtains (cf. (5.10) $)$

$$
V \hat{\Omega}^{-2} V^{*} V \overline{\hat{H}}_{0} V^{*} \chi=\omega \chi
$$

where $\chi \in V \mathfrak{D}$. Defining the bounded invertible multiplication operator $\hat{D}^{-2}:=V \hat{\Omega}^{-2} V^{*}$ and multiplying on the left by $\hat{D}^{2}$ both the members of the equation, one finds

$$
V \overline{\hat{H}}_{0} V^{*} \chi=\hat{D}^{2} \omega \chi
$$


Being $D^{2}=\mathbb{I}_{4}+\mathcal{T}$, where

$$
\mathcal{T}=\left(\begin{array}{cccc}
0 & 0 & 0 & i \alpha(r, \theta) \\
0 & 0 & -i \alpha(r, \theta) & 0 \\
0 & i \alpha(r, \theta) & 0 & 0 \\
-i \alpha(r, \theta) & 0 & 0 & 0
\end{array}\right)
$$

is associated with the multiplication operator $\hat{\mathcal{T}}$ which is bounded and selfadjoint in $\mathcal{H}_{()}$, it follows

$$
\left(V \overline{\hat{H}}_{0} V^{*}-\hat{\mathcal{T}} \omega\right) \chi=\omega \chi,
$$

which is just in the form suitable for variable separation by means of the standard trick. One then obtains the standard form for the separated equations, and formally (compare with equations (5.16), (5.20), (5.21) and (5.22)) the original eigenvalue problem is transformed into the (pseudo-)eigenvalue problem (6.5), in which both the radial part and the angular part are coupled because the angular momentum operator one obtains by variable separation depends on $\omega$ :

$$
\overline{\hat{\mathbb{U}}}_{k \omega}:=\overline{\hat{\mathbb{U}}}_{k}+\hat{\mathbb{V}}_{\omega},
$$

where $\hat{\mathbb{V}}_{\omega}$ is a bounded selfadjoint operator in $L^{2}\left((0, \pi), \frac{\sin (\theta)}{\sqrt{\Delta_{\theta}}} d \theta\right)^{2}$ and is a multiplication operator by

$$
\mathbb{V}_{\omega}=\left(\begin{array}{cc}
0 & -\frac{i \omega a \sin (\theta)}{\sqrt{\Delta_{\theta}}} \\
\frac{i \omega a \sin (\theta)}{\sqrt{\Delta_{\theta}}} & 0
\end{array}\right)=: \omega \mathbb{V}
$$

then also the eigenvalues $\lambda_{k ; j}(\omega)$ of $\overline{\hat{U}}_{k \omega}$ depend on $\omega$. As a consequence, also the radial eigenvalue equation depends on $\omega$ through its dependence on $\lambda_{k ; j}$. See e.g. [19, 24, 12, 13, 14, for the Kerr-Newman case. The following system of coupled eigenvalue equations have to be satisfied simultaneously in $L^{2}\left((0, \pi), \frac{\sin (\theta)}{\sqrt{\Delta_{\theta}}} d \theta\right)^{2}$ and in $L^{2}\left(\left(r_{+}, \infty\right), \frac{r^{2}+a^{2}}{\Delta_{r}} d r\right)^{2}$ respectively:

$$
\overline{\hat{\mathbb{U}}}_{k \omega} S=\lambda S,
$$

and

$$
\overline{\hat{h}}_{k, j} X=\omega X .
$$

We stress again that, the Dirac equation (3.24) in the Chandrasekhar-like variable separation ansatz (3.29) reduces to the couple of equations (6.8) and (6.9) and is equivalent, due to the nature of the operator $\hat{D}^{2}$, to the Hamiltonian eigenvalue equation under the same ansatz.

In order to focus on the relation between the spectral analysis of the Hamiltonian $\overline{\hat{H}}$ and the spectra of the operators $\hat{h}_{k, j}$ which are obtained by variable separation of the pseudo-eigenvalue equation (6.5), we could also heuristically introduce the following trick. Let us consider the 1-parameter family of selfadjoint operators

$$
\left\{\hat{H}_{0}^{(z)}:=V \overline{\hat{H}}_{0} V^{*}-z \hat{\mathcal{T}}\right\}_{z \in \mathbb{R}},
$$

to be defined on a dense domain $D\left(\hat{H}_{0}^{(z)}\right) \subset \mathcal{H}_{()}$, which is easily understood to be independent from $z$. Indeed, $z \hat{\mathcal{T}}$ is a bounded perturbation of $V \overline{\hat{H}}_{0} V^{*}$ and is infinitesimally small with respect to $V \overline{\hat{H}}_{0} V^{*}$ [25] and then, on the domain $V \mathfrak{D}=D\left(V \overline{\hat{H}}_{0} V^{*}\right)=: D\left(\hat{H}_{0}^{(z)}\right), \hat{H}_{0}^{(z)}$ is selfadjoint and defines an analytical family of type (A) according to Kato's definition [26, 27.

Each operator in this family admits an orthogonal decomposition (note that $\overline{\hat{U}}_{k} z=\overline{\hat{\mathbb{U}}}_{k}+z \hat{\mathbb{V}} ;$ cf. (6.7); moreover, the eigenvalues $\lambda_{k ; j}(z)$ of $\overline{\hat{\mathbb{U}}}_{k z}$ depend on $\mathrm{z}$ ):

$$
\hat{H}_{0}^{(z)}=\bigoplus_{k, j} \overline{\hat{h}}_{k, j}^{(z)} \otimes I_{k, j},
$$

and we get

$$
\sigma\left(\hat{H}_{0}^{(z)}\right)=\overline{\bigcup_{k, j} \sigma\left(\overline{\hat{h}}_{k, j}^{(z)}\right)},
$$


and in particular

$$
\sigma_{p}\left(\hat{H}_{0}^{(z)}\right)=\bigcup_{k, j} \sigma_{p}\left(\overline{\hat{h}}_{k, j}^{(z)}\right)
$$

(see e.g. Lemma 7 in [28]).

In order to get a relation between the spectra of this 1-parameter family and the solutions of (6.5), we impose the constraint to include only those $\omega$ such that $\omega \in \sigma\left(\hat{H}_{0}^{(\omega)}\right)$, which implement (6.5).

Note also that, for a non rotating black hole, $a=0$ implies that $\mathcal{T}=0$ and $\Omega^{2}=\mathbb{I}_{4}$. Of course, there is no need to introduce the above 1-parameter family of operators for the study of the spectrum, and $\overline{\hat{H}}=\overline{\hat{H}}_{0}$.

In the following we show that the spectrum of the angular momentum operator $\overline{\hat{U}}_{k \omega}$ is discrete for any $\omega \in \mathbb{R}$. Moreover, we show that in the non-extremal case the radial Hamiltonian $\overline{\hat{h}}_{k, j}$ for any $\lambda_{k ; j}$ has a spectrum is absolutely continuous and coincides with $\mathbb{R}$, and then in the latter case we infer that no eigenvalue of $\overline{\hat{H}}$ exists.

6.1. Spectrum of the operator $\overline{\hat{\mathbb{U}}}_{k \omega}$. We consider the equation $\mathbb{U}_{k \omega} S-\lambda S=0$. As in [21, 29], we look for real solutions for real $\lambda$ and (cf. 21], p. 242) we introduce an analogue to Prüfer transformation in the case of Dirac system. We implement the unitary transformations (5.26) and (5.28) and obtain $R W \overline{\hat{\mathbb{U}}}_{k \omega} W^{*} R^{*}$. Let us define the unitary matrix (cf. [19] for the Kerr-Newman case without magnetic charge)

$$
U:=\frac{1}{\sqrt{2}}\left(\begin{array}{cc}
1 & 1 \\
-1 & 1
\end{array}\right)
$$

and also let us define, thanks to the formal differential expression $R W \mathbb{U}_{k \omega} W^{*} R^{*}$ (see eq. (6.17) below), the following couple of selfadjoint operators:

$$
\begin{aligned}
& D\left(\mathcal{U}_{0}\right)=\left\{\Theta \in L^{2}\left((0, c), \frac{d \theta}{\sqrt{\Delta_{\theta}}}\right)^{2} ; \Theta \text { is locally absolutely continuous; } B(\Theta)=0 ; \mathcal{U}_{0} \Theta \in L^{2}\left((0, c), \frac{d \theta}{\sqrt{\Delta_{\theta}}}\right)^{2}\right\} \\
& \mathcal{U}_{0} \Theta=R W \mathbb{U}_{k \omega} W^{*} R^{*} \Theta, \quad \Theta \in D\left(\mathcal{U}_{0}\right) ; \\
& D\left(\mathcal{U}_{\pi}\right)=\left\{\Theta \in L^{2}\left((c, \pi), \frac{d \theta}{\sqrt{\Delta_{\theta}}}\right)^{2} ; \Theta \text { is locally absolutely continuous; } B(\Theta)=0 ; \mathcal{U}_{\pi} \Theta \in L^{2}\left((c, \pi), \frac{d \theta}{\sqrt{\Delta_{\theta}}}\right)^{2}\right\}, \\
& \mathcal{U}_{\pi} \Theta=R W \mathbb{U}_{k \omega} W^{*} R^{*} \Theta, \quad \Theta \in D\left(\mathcal{U}_{\pi}\right) .
\end{aligned}
$$

$0<c<\pi$ is an arbitrary (regular) point at which the boundary condition $B(\Theta)=\sin (\beta) \Theta_{1}(c)+\cos (\beta) \Theta_{2}(c)=0$, with $\beta \in[0, \pi)$ and with $\Theta(\theta):=\left(\begin{array}{c}\Theta_{1}(\theta) \\ \Theta_{2}(\theta)\end{array}\right)$, is imposed.

One has

$$
U R W \mathbb{U}_{k \omega} W^{*} R^{*} U^{*}=\left(\begin{array}{cc}
0 & \sqrt{\Delta_{\theta}} \partial_{\theta} \\
-\sqrt{\Delta_{\theta}} \partial_{\theta} & 0
\end{array}\right)+M(\theta)
$$

with

$$
M(\theta)=\left(\begin{array}{cc}
\frac{\Xi \sigma(\theta)}{\sqrt{\Delta_{\theta}}} \frac{1}{\sin (\theta)}+\frac{a \omega \sin (\theta)}{\sqrt{\Delta_{\theta}}} & -\mu a \cos (\theta) \\
-\mu a \cos (\theta) & -\frac{\Xi \sigma(\theta)}{\sqrt{\Delta_{\theta}}} \frac{1}{\sin (\theta)}+\frac{a \omega \sin (\theta)}{\sqrt{\Delta_{\theta}}}
\end{array}\right),
$$

where (being $d:=\frac{q_{m} e}{\Xi} \in \mathbb{Z}$ )

$$
\sigma(\theta):=d \cos (\theta)-\left(n+\frac{1}{2}\right), \quad d, n \in \mathbb{Z} .
$$

We can rewrite $U R W \mathbb{U}_{k \omega} W^{*} R^{*} U^{*}$ in the following form:

$$
\mathfrak{R}^{-1}(\theta)\left[\left(\begin{array}{cc}
0 & \partial_{\theta} \\
-\partial_{\theta} & 0
\end{array}\right)+\mathfrak{R}(\theta)\left(\begin{array}{cc}
\frac{\Xi \sigma(\theta)}{\sqrt{\Delta_{\theta}}} \frac{1}{\sin (\theta)}+\frac{a \omega \sin (\theta)}{\sqrt{\Delta_{\theta}}} & -\mu a \cos (\theta) \\
-\mu a \cos (\theta) & -\frac{\Xi \sigma(\theta)}{\sqrt{\Delta_{\theta}}} \frac{1}{\sin (\theta)}+\frac{a \omega \sin (\theta)}{\sqrt{\Delta_{\theta}}}
\end{array}\right)\right],
$$

where

$$
\mathfrak{R}^{-1}(\theta)=\left(\begin{array}{cc}
\sqrt{\Delta_{\theta}} & 0 \\
0 & \sqrt{\Delta_{\theta}}
\end{array}\right)
$$


As in [21, 29], we can define

$$
G(\theta, \lambda)=\lambda \Re(\theta)-M(\theta),
$$

and

$$
\Theta(\theta)=\bar{\rho}(\theta)\left(\begin{array}{c}
\cos \eta(\theta) \\
\sin \eta(\theta)
\end{array}\right)
$$

where

$$
\bar{\rho}(\theta)=\sqrt{\Theta_{1}^{2}(\theta)+\Theta_{2}^{2}(\theta)}
$$

and

$$
\eta(\theta)= \begin{cases}\arctan \frac{\Theta_{2}(\theta)}{\Theta_{1}(\theta)} & \text { for } \Theta_{1}(\theta) \neq 0 \\ \operatorname{arccot} \frac{\Theta_{1}(\theta)}{\Theta_{2}(\theta)} & \text { for } \Theta_{2}(\theta) \neq 0\end{cases}
$$

are defined for real solutions of the eigenvalue equation and are absolutely continuous [29]. Then following [21, 29] one obtains the following differential equation for $\eta(\theta)$ :

$$
\frac{d}{d \theta} \eta(\theta, \lambda)=H(\theta, \eta(\theta), \lambda)
$$

where

$$
H(\theta, \eta(\theta), \lambda):=\left(G(\theta, \lambda)\left(\begin{array}{c}
\cos \eta(\theta) \\
\sin \eta(\theta)
\end{array}\right) \mid\left(\begin{array}{c}
\cos \eta(\theta) \\
\sin \eta(\theta)
\end{array}\right)\right)
$$

and with $(\cdot \mid \cdot)$ here we mean the usual Euclidean product in $\mathbb{C}^{2}$. One then finds

$$
\begin{aligned}
H(\theta, \eta(\theta), \lambda) & =\frac{\lambda}{\sqrt{\Delta_{\theta}}}+(2 a \mu \cos (\theta)) \sin (\eta(\theta)) \cos (\eta(\theta)) \\
& +\left[\frac{\Xi \sigma(\theta)}{\sqrt{\Delta_{\theta}}} \frac{1}{\sin (\theta)}+\frac{a \omega \sin (\theta)}{\sqrt{\Delta_{\theta}}}\right]\left(\sin ^{2}(\eta(\theta))-\cos ^{2}(\eta(\theta))\right) .
\end{aligned}
$$

Note that the function $H(\theta, t, \lambda)$ is smooth for any $(\theta, t, \lambda) \in(0, \pi) \times \mathbb{R} \times \mathbb{R}$. We consider first the case of the operator $\mathcal{U}_{\pi}$. Let us define as in 21$]$

$$
\begin{aligned}
n_{+}\left(\lambda_{1}, \lambda_{2}\right) & =\liminf _{\theta \rightarrow \pi^{-}} \frac{1}{\pi}\left(\eta\left(\theta, \lambda_{2}\right)-\eta\left(\theta, \lambda_{1}\right)\right) \\
n_{-}\left(\lambda_{1}, \lambda_{2}\right) & =\limsup _{\theta \rightarrow \pi^{-}} \frac{1}{\pi}\left(\eta\left(\theta, \lambda_{2}\right)-\eta\left(\theta, \lambda_{1}\right)\right) \\
M\left(\lambda_{1}, \lambda_{2}\right) & =\operatorname{dim}\left(E\left(\lambda_{2}\right)-E\left(\lambda_{1}\right)\right),
\end{aligned}
$$

where $E(\lambda)$ is the spectral resolution of $\mathcal{U}_{\pi}$. It holds $n_{-}\left(\lambda_{1}, \lambda_{2}\right)-2 \leq M\left(\lambda_{1}, \lambda_{2}\right) \leq n_{+}\left(\lambda_{1}, \lambda_{2}\right)+2$. In particular, $\lambda$ belongs to the essential spectrum iff for every $\epsilon>0$ it holds

$$
n_{+}(\lambda-\epsilon, \lambda+\epsilon)=\infty
$$

(cf. [21], p. 248). We now prove the following result.

Theorem 4. The essential spectrum of $\overline{\hat{U}}_{k \omega}$ is empty.

Proof. We start by considering $\mathcal{U}_{\pi}$. Our aim is to show that the function $\eta(\theta, \lambda)$ is a bounded function for any finite $\lambda$. As a consequence, the essential spectrum of $\mathcal{U}_{\pi}$ is empty. With this aim, we assume that $\eta$ is unbounded. We show that this assumption leads to a contradiction both if $\eta$ has no upper bound as $\theta \rightarrow \pi^{-}$ and if $\eta$ has no lower bound as $\theta \rightarrow \pi^{-}$.

We first note that the function

$$
\zeta(\theta):=\frac{\sigma(\theta)}{\sqrt{\Delta_{\theta}}} \frac{1}{\sin (\theta)}
$$

in a suitable left neighborhood of $\pi$ is either increasing or decreasing according to the sign of $\sigma(\theta)$ at $\pi$ (its derivative leading term is $-\cos (\theta) \frac{\sigma(\theta)}{\sqrt{\Delta_{\theta}}} \frac{1}{\sin ^{2}(\theta)}$ in such a neighborhood). Let us assume that $\sigma(\pi)>0$. Then, for 
$\theta_{0}<\theta<\pi$ the function $\sigma(\theta)$ is positive and for $\theta_{1}<\theta<\pi$, where $\theta_{0} \leq \theta_{1}$, the function $\zeta$ is increasing without upper bound. Then we can choose a $\theta_{2}>\theta_{1}$ such that for any $\theta_{2}<\theta<\pi$ it holds

$$
\frac{\Xi \sigma(\theta)}{\sqrt{\Delta_{\theta}}} \frac{1}{\sin (\theta)}+\frac{a \omega \sin (\theta)}{\sqrt{\Delta_{\theta}}}>\left|\frac{\lambda}{\sqrt{\Xi}}\right|+2|a \mu| .
$$

If $\eta$ has no upper bound, then there exists a $\theta_{3}>\theta_{2}$ such that

$$
\eta\left(\theta_{3}, \lambda\right)=p \pi \quad \text { for } p \in \mathbb{Z}
$$

and

$$
\eta(\theta, \lambda)>\eta\left(\theta_{3}, \lambda\right)
$$

for $\theta_{3}<\theta<\theta_{4}<\pi$, i.e. in a suitable right neighborhood of $\theta_{3}$ the function $\eta(\theta, \lambda)$ has to be increasing; if $\eta$ has no lower bound, then there exists a $\theta_{5}>\theta_{2}$ such that

$$
\eta\left(\theta_{5}\right)=\left(p+\frac{1}{2}\right) \pi \quad \text { for } p \in \mathbb{Z}
$$

and

$$
\eta(\theta, \lambda)<\eta\left(\theta_{5}, \lambda\right)
$$

for $\theta_{5}<\theta<\theta_{6}<\pi$. In both cases a contradiction is achieved, indeed the function $H(\theta, \eta(\theta), \lambda)$ is negative in a suitable neighborhood of $\theta_{3}$ in the former case because of (6.31):

$$
H\left(\theta_{3}, \eta\left(\theta_{3}\right), \lambda\right)=\frac{\lambda}{\sqrt{\Delta_{\theta_{3}}}}-\left[\Xi \frac{\sigma\left(\theta_{3}\right)}{\sqrt{\Delta_{\theta_{3}}}} \frac{1}{\sin \left(\theta_{3}\right)}+\frac{a \omega \sin \left(\theta_{3}\right)}{\sqrt{\Delta_{\theta_{3}}}}\right]<0 .
$$

Then $\eta$ cannot be unbounded from above (cf. (6.25)).

In the latter case one finds $H\left(\theta_{5}, \eta\left(\theta_{5}\right), \lambda\right)>0$ and then $\eta$ cannot be unbounded from below. In the case $\sigma(\pi)<0$, one gets the same contradiction in an analogous way.

Analogously, one can conclude that $\sigma_{e}\left(\mathcal{U}_{0}\right)=\emptyset$. The decomposition method 21] ensures that $\sigma_{e}\left(U R W \overline{\hat{U}}_{k \omega} W^{*} R^{*} U^{*}\right)=$ $\sigma_{e}\left(\mathcal{U}_{0}\right) \cup \sigma_{e}\left(\mathcal{U}_{\pi}\right)$, and then we can conclude that the spectrum of $\overline{\mathbb{U}}_{k \omega}$ is discrete. On the grounds of theorem 10.8 in [21], we can also conclude that the spectrum is simple.

See also Appendix $B$ for an alternative proof. Note that for $\omega=0$ Theorem 4 implies that the spectrum of $\overline{\mathbb{U}}_{k}$ is discrete.

6.2. Spectrum of the operator $\overline{\hat{h}}_{k, j}$. In order to study the spectral properties of $\overline{\hat{h}}_{k, j}$, we introduce, as in the previous subsection, two auxiliary selfadjoint operators $\hat{h}_{h o r}$ and $\hat{h}_{\infty}$ :

$$
\begin{aligned}
& D\left(\hat{h}_{h o r}\right)=\left\{X \in L_{\left(r_{+}, r_{0}\right)}^{2}, X \text { is locally absolutely continuous; } B(X)=0 ; \hat{h}_{h o r} X \in L_{\left(r_{+}, r_{0}\right)}^{2}\right\}, \\
& \hat{h}_{h o r} X=h_{k, j} X ; \\
& D\left(\hat{h}_{\infty}\right)=\left\{X \in L_{\left(r_{0}, \infty\right)}^{2}, X \text { is locally absolutely continuous; } B(X)=0 ; \hat{h}_{\infty} X \in L_{\left(r_{0}, \infty\right)}^{2}\right\} \\
& \hat{h}_{\infty} X=h_{k, j} X .
\end{aligned}
$$

$r_{0}$ is an arbitrary point with $r_{+}<r_{0}<\infty$, at which the boundary condition $B(X):=\sin (\beta) X_{1}\left(r_{0}\right)+$ $\cos (\beta) X_{2}\left(r_{0}\right)=0$, with $X(r):=\left(\begin{array}{c}X_{1}(r) \\ X_{2}(r)\end{array}\right)$ and with $\beta \in[0, \pi)$ is imposed. We also have defined $L_{\left(r_{+}, r_{0}\right)}^{2}:=$ $L^{2}\left(\left(r_{+}, r_{0}\right), \frac{r^{2}+a^{2}}{\Delta_{r}} d r\right)^{2}$ and $L_{\left(r_{0}, \infty\right)}^{2}:=L^{2}\left(\left(r_{0}, \infty\right), \frac{r^{2}+a^{2}}{\Delta_{r}} d r\right)^{2}$. Note that we omit the indices $k, j$ for these operators.

We first show that $\hat{h}_{\infty}$ has discrete spectrum and that in the non-extremal case $\hat{h}_{h o r}$ has absolutely continuous spectrum, and then we deduce qualitative spectral properties for $\overline{\hat{h}}_{k, j}$.

On the grounds of the analysis in [30, 31, we can conclude that the spectrum of $\overline{\hat{h}}_{k, j}$ is absolutely continuous in the non-extremal case. As to the extremal manifold, we limit ourselves to point out that $\sigma_{e}\left(\overline{\hat{h}}_{\infty}\right)=\emptyset$ holds true, too; a weaker conclusion can instead be stated about the spectrum of $\hat{h}_{h o r}$ : it is absolutely continuous in $\mathbb{R}-\varphi_{+}$. 
We introduce the tortoise coordinate

$$
\frac{d x}{d r}=\frac{r^{2}+a^{2}}{\Delta_{r}}
$$

and choose the integration constant in such a way that $r \in\left(r_{+}, \infty\right)$ iff $x \in(-\infty, 0)$. We also point out that, for $r \rightarrow \infty$, i.e. for $x \rightarrow 0^{-}$one finds $r \sim-\frac{l^{2}}{x}$. We get

$$
\hat{h}_{k, j}=\left(\begin{array}{cc}
0 & \partial_{x} \\
-\partial_{x} & 0
\end{array}\right)+V(r(x)) \text {. }
$$

We consider $\hat{h}_{\infty}=\left.\hat{h}_{k, j}\right|_{\left[x\left(r_{0}\right), 0\right)}$. We introduce the Prüfer-like transformation as in the case of the angular momentum operator

$$
\bar{\rho}(x)=\sqrt{X_{1}^{2}(x)+X_{2}^{2}(x)}
$$

and

$$
\eta(x)= \begin{cases}\arctan \frac{X_{2}(x)}{X_{1}(x)} & \text { for } X_{1}(x) \neq 0 \\ \operatorname{arccot} \frac{X_{1}(x)}{X_{2}(x)} & \text { for } X_{2}(x) \neq 0\end{cases}
$$

We can also define

$$
G(x, \omega):=\omega \mathbb{I}-V(r(x)),
$$

and obtain the differential equation

$$
\frac{d}{d x} \eta(x, \omega)=H(x, \eta(x), \omega)
$$

where

$$
H(x, \eta(x), \omega):=\left(G(x, \omega)\left(\begin{array}{c}
\cos \eta(x) \\
\sin \eta(x)
\end{array}\right) \mid\left(\begin{array}{c}
\cos \eta(x) \\
\sin \eta(x)
\end{array}\right)\right) .
$$

One then finds (the dependence of $r$ on $x$ is left implicit)

$$
\begin{aligned}
H(x, \eta(x), \omega) & =\omega-\frac{a \Xi k+e q_{e} r}{r^{2}+a^{2}}-\frac{2 \lambda_{k ; j} \sqrt{\Delta_{r}}}{r^{2}+a^{2}} \sin (\eta(x, \omega)) \cos (\eta(x, \omega)) \\
& +\frac{\mu r \sqrt{\Delta_{r}}}{r^{2}+a^{2}}\left(\sin ^{2}(\eta(x, \omega))-\cos ^{2}(\eta(x, \omega)) .\right.
\end{aligned}
$$

Note that the function $H(x, s, \omega)$ is smooth for $(x, s, \omega) \in(-\infty, 0) \times \mathbb{R} \times \mathbb{R}$.

Lemma 2. $\sigma_{e}\left(\hat{h}_{\infty}\right)=\emptyset$.

Proof. The leading term in the potential is proportional to the mass $\mu$ and is monotonically increasing in a suitable left neighborhood $x_{0}<x<0$ of $x=0$. We can also find an $x_{1} \in\left[x_{0}, 0\right)$ such that for $x_{1}<x<0$ one gets

$$
\mu \frac{r \sqrt{\Delta_{r}}}{r^{2}+a^{2}}>|\omega|+\frac{\left|a \Xi k+e q_{e} r\right|}{r^{2}+a^{2}}+2\left|\lambda_{k ; j}\right| \frac{\sqrt{\Delta_{r}}}{r^{2}+a^{2}} .
$$

If $\eta$ is not bounded from above, we can find an $x_{2} \in\left(x_{1}, 0\right)$ such that $\eta\left(x_{2}, \omega\right)=p \pi$, with $p \in \mathbb{Z}$, and $\eta(x, \omega)>\eta\left(x_{2}, \omega\right)$ for $x_{2}<x<x_{3}<0$. If $\eta$ is not bounded from below, we can find an $x_{4} \in\left(x_{1}, 0\right)$ such that $\eta\left(x_{4}, \omega\right)=\left(q+\frac{1}{2}\right) \pi$, with $q \in \mathbb{Z}$, and $\eta(x, \omega)<\eta\left(x_{4}, \omega\right)$ for $x_{4}<x<x_{5}<0$. But due to (6.46) $H(x, \eta(x), \omega)$ would be negative in a neighbourhood of $x_{2}$ and it would be positive in a neighbourhood of $x_{4}$, against the assumption of an unbounded $\eta$. As a consequence, $\eta$ has to be bounded, and then the essential spectrum of $\hat{h}_{\infty}$ is empty.

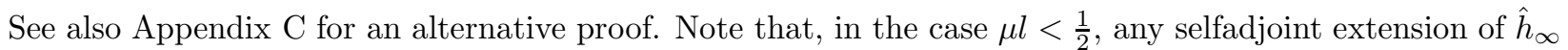
obtained by imposing separated boundary conditions at $r_{0}$ and at $r=\infty$ still has discrete spectrum [21]. It is also remarkable that $\sigma_{e}\left(\hat{h}_{\infty}\right)=\emptyset$ is not verified in the standard Kerr-Newman case.

As to the spectral properties of $\hat{h}_{h o r}$, a suitable change of coordinates consists in introducing a tortoise-like coordinate defined by eqn. (5.40). It is then easy to show that the following result holds. 
Lemma 3. $\sigma_{a c}\left(\hat{h}_{h o r}\right)=\mathbb{R}$ in the non-extremal case.

Proof. The hypotheses of theorem 1 p. 185 of [30] are verified. Equivalently in our case we can appeal to theorem 16.7 of [21, and we find that the spectrum of $\hat{h}_{h o r}$ is absolutely continuous in $\mathbb{R}-\left\{\varphi_{+}\right\}$. This can be proved as follows. Let us write the potential $V(r(y))$ in (5.41)

$$
V(r(y))=\left(\begin{array}{cc}
\varphi_{+} & 0 \\
0 & \varphi_{+}
\end{array}\right)+P_{2}(r(y))
$$

which implicitly defines $P_{2}(r(y))$. The first term on the left of 6.47 is of course of bounded variation; on the other hand, $\left|P_{2}(r(y))\right| \in L^{1}(c, \infty)$, with $c \in(0, \infty)$. As a consequence, the hypotheses of theorem 16.7 in [21] are trivially satisfied, and one finds that the spectrum of $\hat{h}_{h o r}$ is absolutely continuous in $\mathbb{R}-\left\{\varphi_{+}\right\}$.

We have only to exclude that $\varphi_{+}$is not an eigenvalue of $\hat{h}_{h o r}$ (and of the radial Hamiltonian). As in the Kerr-Newman case (cf. e.g. [12]), one needs simply to replace $\omega$ with $\varphi_{+}$and study the asymptotic behavior of the solutions of the linear system

$$
X^{\prime}=\left(\begin{array}{cc}
-\lambda_{k ; j} \frac{\sqrt{\Delta_{r}}}{r^{2}+a^{2}} & \varphi_{+}-\frac{1}{r^{2}+a^{2}}\left(a \Xi k+e q_{e} r-\mu r \sqrt{\Delta_{r}}\right) \\
\frac{1}{r^{2}+a^{2}}\left(a \Xi k+e q_{e} r+\mu r \sqrt{\Delta_{r}}\right)-\varphi_{+} & \lambda_{k ; j} \frac{\sqrt{\Delta_{r}}}{r^{2}+a^{2}}
\end{array}\right) X=: \bar{R}(r(y)) X,
$$

where $r=r(y)$ and where the prime indicates the derivative with respect to $y$. One easily realizes that in the non-extremal case

$$
\int_{c}^{\infty} d y|\bar{R}(r(y))|<\infty
$$

and then according to the Levinson theorem (see e.g. [32, Theorem 1.3.1 p.8) one can find two linearly independent asymptotic solutions as $y \rightarrow \infty$ whose leading order is given by $X_{I}=\left(\begin{array}{l}1 \\ 0\end{array}\right)$ and $X_{I I}=\left(\begin{array}{l}0 \\ 1\end{array}\right)$. As a consequence no normalizable solution of the equation 6.48 can exists, and then $\varphi_{+}$cannot be an eigenvalue.

Note that in the non-extremal case, theorem 16.7 in [21] applies also to $\overline{\hat{h}}_{k, j}$. The following result holds:

Theorem 5. $\sigma_{a c}\left(\overline{\hat{h}}_{k, j}\right)=\mathbb{R}$ in the non-extremal case. Moreover, the spectrum is simple.

Proof. As a consequence of Lemma 2 and Lemma 3 (cf. also remark (2), pp. 211-212 of [30]), we can conclude that the spectrum of $\hat{h}_{k, j}$ is absolutely continuous and coincides with $\mathbb{R}$. On the grounds of theorem 10.8 in 21, we can also conclude that the spectrum is simple.

As to the extremal case, we limit ourselves to state the following result.

Lemma 4. The spectrum of $\overline{\hat{h}}_{k, j}$ is absolutely continuous in $\mathbb{R}-\left\{\varphi_{+}\right\}$in the extremal case.

Proof. We refer to theorem 1 p. 185 in 30. By using the tortoise coordinate (5.40) we rewrite the radial eigenvalue equation (6.9) in the form

$$
X^{\prime}=\left(\begin{array}{cc}
p(r(y)) & \omega+p_{2}(r(y)) \\
-\omega-p_{1}(r(y)) & -p(r(y))
\end{array}\right) X,
$$

where the prime stays for the derivative with respect to $y, p(r):=-\frac{\lambda_{k ; j} \sqrt{\Delta_{r}}}{r^{2}+a^{2}}, p_{1}(r):=-\frac{1}{r^{2}+a^{2}}\left(a \Xi k+e q_{e} r+\right.$ $\left.\mu r \sqrt{\Delta_{r}}\right)$ and $p_{2}(r):=-\frac{1}{r^{2}+a^{2}}\left(a \Xi k+e q_{e} r-\mu r \sqrt{\Delta_{r}}\right)$.

Following the notation in [30], we define $p(r(y)):=\Delta_{1}, p_{11}(r(y)):=p_{1}(r(y))$ and $p_{21}(r(y)):=p_{2}(r(y))$. One has $p_{11}(r(y)) \rightarrow-\varphi_{+}$and $p_{21}(r(y)) \rightarrow-\varphi_{+}$as $y \rightarrow \infty$; moreover, $\Delta_{1}(r(y)) \rightarrow 0$ as $y \rightarrow \infty$ and $p^{\prime}{ }_{11}, p^{\prime}{ }_{21}, \Delta^{\prime}{ }_{1} \in L^{1}\left[r_{0}, \infty\right)$, with $r_{+}<r_{0}<\infty$. Then the hypotheses of theorem 1 p. 185 in [30] are satisfied, and then the spectrum of $\hat{h}_{k, j}$ is absolutely continuous in $\mathbb{R}-\left\{\varphi_{+}\right\}$. Cf. also Remark (1) p. 211 in [30.

The analysis of the point $\left\{\varphi_{+}\right\}$is more involved than in the non-extremal case and is deferred to further studies.

The above result allow us to conclude also that the following result holds for the essential spectrum of the radial Hamiltonian: 
Corollary 1. $\sigma_{e}\left(\overline{\hat{h}}_{k, j}\right)=\mathbb{R}$ both for the non-extremal case and for the extremal one.

Proof. In the non-extremal case, the result follows from Theorem 5 . In the extremal one, is a consequence of Lemma 4.

Both these results can also be achieved by using Theorem 16.6 in [21. Indeed, let us consider the Hamiltonian (5.41) and, in order to match Weidmann's conditions, let us introduce the unitary operator $\begin{array}{ll}Z:= \\ 1\end{array}\left(\begin{array}{c}0 \\ 1\end{array}\right)$. Then let us consider $Z \hat{h}_{h o r} Z^{*}$, whose formal expression is

$$
Z h_{h o r} Z^{*}=\left(\begin{array}{cc}
0 & \partial_{y} \\
-\partial_{y} & 0
\end{array}\right)+\bar{P}(y)
$$

and define $\bar{P}_{0}:=\lim _{y \rightarrow \infty} \bar{P}(y)=\left(\begin{array}{cc}\varphi_{+} & 0 \\ 0 & \varphi_{+}\end{array}\right)$. According to Theorem 16.6 in [21], if

$$
\lim _{y \rightarrow \infty} \frac{1}{y} \int_{c}^{y} d t\left|\bar{P}(t)-\bar{P}_{0}\right|=0
$$

where $|\cdot|$ stays for any norm for matrices in $\mathbb{C}^{2 \times 2}$, then $\mathbb{R}-\left\{\varphi_{+}\right\} \subset \sigma_{e}\left(\hat{h}_{h o r}\right)$, which implies $\sigma_{e}\left(\hat{h}_{h o r}\right)=\mathbb{R}$. By using e.g. the Euclidean norm, it is easy to show that in the non-extremal case it holds $\left|\int_{c}^{\infty} d t\right| \bar{P}(t)-\bar{P}{ }_{0} \|<\infty$, and then (6.52) is implemented. In the extremal case, the integral is divergent as $y \rightarrow \infty$ but De l'Hospital's rule allows still to conclude that (6.52) is implemented. Cf. also [4] for the standard Kerr-Newman case and [5] for the Reissner-Nordström-AdS case.

6.3. Absence of time-periodic normalizable solutions. For the non extremal case, the spectral analysis carried out in the previous subsections allows to conclude that on the given black hole background the Dirac equation does not admit any normalizable time-periodic solution. Cf. 11] and [12] for the Kerr-Newman case. Both the hypothesis and the proof of such a theorem are completely analogous to the ones relative to the nonextremal Kerr-Newman black hole case appearing in [12, theorem IV.5, with simple and obvious replacements. We can limit ourselves to observe that, given the Dirac equation in its Hamiltonian form (4.1), it is possible to obtain solutions which are both normalizable and time-periodic if and only if the point spectrum of the Hamiltonian $\overline{\hat{H}}$ is non-empty. For the sake of completeness we show that the aforementioned remark holds true. We are indebted to Franco Gallone (Università di Milano) for providing us a rigorous proof of it.

Remark 1. Let $\hat{G}$ be a selfadjoint operator in a Hilbert space $\mathcal{H}$ and let $P$ the projection valued measure of $\hat{G}$; let $\hat{U}$ be the 1-parameter strongly continuous group generated by $\hat{G}$. There exist $T \in \mathbb{R}-\{0\}$ and $\psi \in \mathcal{H}-\{0\}$ such that $\hat{U}_{t+T} \psi=\hat{U}_{t} \psi$ for each $t \in \mathbb{R}$ iff the point spectrum of $\hat{G}$ is non-empty.

Proof. For $T \in \mathbb{R}-\{0\}$ and $\psi \in \mathcal{H}, \hat{U}_{t+T} \psi=\hat{U}_{t} \psi$ is equivalent to $\hat{U}_{T} \psi=\psi$, which amounts to the condition

$$
\int_{\mathbb{R}}\left|\mathrm{e}^{i \lambda T}-1\right|^{2} d \mu_{\psi}^{(P)}(\lambda)=0
$$

(where for each $E$ contained in the Borel $\sigma$-algebra $\mathcal{B}$ in $\mathbb{R}$, the measure $\mu_{\psi}^{(P)}$ is defined by $\left.\mu_{\psi}^{(P)}(E):=\|P(E) \psi\|^{2}\right)$. The above condition is implemented iff $\mathrm{e}^{i \lambda T}=1 \mu_{\psi}$-a.e., which amounts to $\mu_{\psi}^{(P)}\left(\left\{\frac{2 \pi n}{T}\right\}_{n \in \mathbb{Z}}\right)=\|\psi\|^{2}$, i.e. $P\left(\left\{\frac{2 \pi n}{T}\right\}_{n \in \mathbb{Z}}\right) \psi=\psi$. Then there exists a $\psi \neq 0$ such that $\hat{U}_{t+T} \psi=\hat{U}_{t} \psi$ for every $t \in \mathbb{R}$ iff $P\left(\left\{\frac{2 \pi n}{T}\right\}_{n \in \mathbb{Z}}\right) \neq 0$, and the latter condition holds iff there exists $n_{0} \in \mathbb{Z}$ such that $P\left(\left\{\frac{2 \pi n_{0}}{T}\right\}\right) \neq 0$, i.e. such that $\frac{2 \pi n_{0}}{T} \in \sigma_{p}(\hat{G})$.

In the case at hand, $\hat{G}$ is the Hamiltonian operator $\overline{\hat{H}}$ and $t$ is the time. For a non-extremal Kerr-NewmanAdS black hole, we have that the point spectrum of $\overline{\hat{H}}$ is empty (cf. also Lemma 5.3 in [14] and Proposition 7.1 in 15 for then Kerr-Newman case) and then no time-periodic and normalizable solution of the Dirac equation is allowed. From a physical point of view, this fact means that no quantum mechanical solution equivalent to a classical closed orbit exists. Cf. [10, 11]. 


\section{Conclusions}

We have considered the Dirac equation on the universal covering of a Kerr-Newman-AdS black hole background. The presence of a magnetic charge has been allowed, and the Hamiltonian form of the Dirac equation has been obtained. Then, we have shown that Theorem 1 holds true, and then we have studied essential selfadjointness properties of the Hamiltonian $\hat{H}$ on $C_{0}^{\infty}\left(\left(r_{+}, \infty\right) \times S^{2}\right)^{4}$ through the equivalent analysis on $\hat{H}_{0}$. Variable separation has been performed and we have shown that in presence of a magnetic charge the Dirac quantization condition $\frac{q_{m} e}{\Xi} \in \mathbb{Z}$ is necessary and sufficient for ensuring essential selfadjointness of $\mathbb{U}_{k}$ on $C_{0}^{\infty}(0, \pi)^{2}$ for any

$k \in \mathbb{Z}+\frac{1}{2}$. Moreover, $\mu l \geq \frac{1}{2}$ has to be implemented in order to obtain essential selfadjointness of the radial Hamiltonian $\hat{h}_{k, j}$ on $C_{0}^{\infty}\left(r_{+}, \infty\right)^{2}$. This is also the condition one finds on an Anti-de Sitter background.

Furthermore, we have taken into account some spectral properties of the Hamiltonian for $\mu l \geq \frac{1}{2}$. Qualitative spectral analysis has allowed us to conclude that $\sigma_{e}\left(\hat{h}_{\infty}\right)=\emptyset$, in contrast to what happens in the (asymptotically flat) Kerr-Newman case, and also that no time-periodic and normalizable solution of the Dirac equation is allowed on the given non-extremal black hole background. The latter conclusion is in agreement with the analogous result for black hole of the Kerr-Newman family and more in general for axi-symmetric black holes which are asymptotically flat [11, 12. Moreover, in the case $\mu l<\frac{1}{2}$ this holds true also for selfadjoint extensions $\hat{T}_{H}$ obtained by imposing local boundary conditions at infinity. Cf. the comment following Lemma 2 .

The implementation of a second-quantization formalism and the analysis of the mechanism allowing both the discharge and the loss of angular momentum by the black hole by means of quantum effects [3, 4, deserve future investigations.

\section{Acknowledgments}

We are indebted to Franco Gallone (Università degli Studi di Milano) for several discussions and fruitful comments and for a rigorous proof of Remark 1. We thanks also Aldo Treves, Andrea Posilicano and Stefano Pigola (Università dell'Insubria) for discussions and remarks.

\section{Appendix A. Essential SElfadjointness of $\hat{\mathbb{U}}$}

For the sake of completeness, we discuss in detail the essential selfadjointness conditions for the angular momentum operator $\mathbb{U}$. Let us introduce $d:=\frac{q_{m} e}{\Xi}$ and also the sets

$$
I_{0}:=(-1+d, d),
$$

which is such that for $n \in I_{0}$ the limit circle case [21] is implemented at $\theta=0$, and

$$
I_{\pi}:=(-1-d,-d),
$$

which is such that for $n \in I_{\pi}$ the limit circle case is implemented at $\theta=\pi$. Essential selfadjointness for a generic $n \in \mathbb{R}$ is implemented for

$$
n \in \mathbb{R}-\left(I_{0} \cup I_{\pi}\right) .
$$

Condition (A.3) for $|d| \leq \frac{1}{2}$ amounts to

$$
n \in(-\infty,-1-|d|] \cup[|d|, \infty) .
$$

If $|d|>\frac{1}{2}$, condition (A.3) amounts to

$$
n \in(-\infty,-1-|d|] \cup[-|d|,-1+|d|] \cup[|d|, \infty) .
$$

Now we make use of the fact that $n \in \mathbb{Z}$ in our case. For $d \in \mathbb{Z}$, essential selfadjointness is implemented without restrictions. If $|d|:=[|d|]+\zeta$, where $\zeta \in(0,1)$, then essential selfadjointness is implemented for $n \in \mathbb{Z}-\{-1-[|d|],[|d|]\}$, which requires further analysis. We can note that choosing $d \in \mathbb{Z}$ amounts to the Dirac quantization condition in the stronger form which was considered as mandatory by Schwinger in his model for a relativistic quantum field theory for fermionic matter in presence of a magnetic charge 33] and in which only integer values are allowed instead of semi-integer values. See also 34. This quantization rule is confirmed also by a construction à la Wu and Yang [35. See e.g. [36] (formula (11) therein and its consequences for $a=0$ on the charge quantization, which corresponds to our case).

For the sake of completeness, we point out that semi-integer values for $d$ would introduce the necessity of 
implementing suitable boundary conditions at $\theta=0$ or at $\theta=\pi$ for $\mathbb{U}_{k}$ for special values of $k$, which are such that the defect indices of $\mathbb{U}_{k}$ are $(1,1)$. Let us put $|d|=l+\frac{1}{2}$, with $l \in \mathbb{N}$. Then we find that $n=-l-1$ and $n=l$ do not satisfy the essential selfadjointness conditions indicated above. Being $I_{0} \cap I_{\pi}=\emptyset$ for $|d|=l+\frac{1}{2}$, which means that the limit circle case occurs only at one of the extremes of $(0, \pi)$, one finds that the partial wave operators $\mathbb{U}_{-l-\frac{1}{2}}$ and $\mathbb{U}_{l+\frac{1}{2}}$ have defect indices $(1,1)$. This would introduce an "asymmetric" treatment for a couple of partial waves labeled by $k$ with respect to all the other ones which do not require boundary conditions. This "asymmetry" would not be justified by any physical argument, being the singular behavior at $\theta=0$ or at $\theta=\pi$ only due to a bad behavior of the chart one is forced to introduce if a single-chart description of the 1-form connection $A$ is adopted, as in our case; then the following choice is taken into account: a core for the extension is identified with $C_{0}^{\infty}(0, \pi)^{2}+L\left\{w_{+}\right\}$[21, where $w_{+}$is such that near the point $\theta_{0}\left(\theta_{0}=0\right.$ or $\left.\theta_{0}=\pi\right)$ at which the limit circle case occurs one has $w_{+} \sim u_{s}\left(u_{s} \sim\left(\theta-\theta_{0}\right)^{s}\right.$ being the fundamental solution of the equation $\mathbb{U}_{k} u-\lambda u=0$ near $\theta_{0}$ with $s=\max ( \pm \nu)$ if $\theta_{0}=0$, cf. (5.31), or $s=\max \left( \pm \rho_{0}\right)$ if $\theta_{0}=\pi$, cf. (5.36) ), and $w_{+} \sim 0$ at the other extreme. This is the only choice ensuring, together with the condition $\mu l \geq \frac{1}{2}$, that $\hat{H}_{0}$ is essentially selfadjoint on $C_{0}^{\infty}\left(\left(r_{+}, \infty\right) \times S^{2}\right)^{4}$ (lack of smoothness at $\theta_{0}$ occurs for any other choice). It must be pointed out that another choice of the gauge would be possible and that it would lead to different conditions. At least almost everywhere, gauge equivalence of the given $A$ with

$$
A+b\left(\frac{q_{m}}{\rho \sqrt{\Delta \theta} \sin \theta} e^{1}-\frac{q_{m} a}{\rho \sqrt{\Delta_{r}}} e^{0}\right)
$$

can be shown for any value of the real constant $b$. Then the replacements $q_{m} \cos \theta \mapsto q_{m}(\cos \theta-b)$ and $q_{e} r \mapsto q_{e} r+b q_{m} a$ occur. Essential selfadjointness properties of $\hat{h}_{k, j}$ remain unaltered for any $b$. Instead, if for example one chooses $b=1$, essential selfadjointness properties of $\mathbb{U}_{k}$ are affected in an evident manner: this choice, which corresponds to the usual choice introduced since the seminal paper by Dirac, is such that all problems are shifted to $\theta=\pi$ and conditions (A.4) and (A.5) are replaced by the essential selfadjointness condition

$$
n \in(-\infty,-1-2 d] \cup[-2 d, \infty)
$$

Then, semi-integer values of the magnetic charge ensure essential selfadjointness too, in agreement with the general form of the Dirac quantization condition. We are not aware of a solution of the dichotomy between the physical situation represented by the Dirac string and the Schwinger one but for the explanation given in 37]: in fact for a fixed magnetic field the infinite singularity line embodied by the choice of the Schwinger potential is associated with a monopole of double strength with respect to the one which is associated with the Dirac semi-infinite singularity line (a double flux is generated by the former with respect to the latter). Hence a factor 2 appears. In other terms, the relation $q_{m}^{\text {Schwinger }}=2 q_{m}^{\text {Dirac }}$ should occur. Then it should be also true that the validity of the aforementioned gauge equivalence is only almost everywhere (smooth part) and that the singular part cannot be gauge-equivalent due to the different behavior of a semi-infinite string with respect to an infinite one. This interpretation would be also corroborated by the fact that, as it is evident, the corresponding transformation of the Hamiltonian cannot be implemented by means of a unitary transformation (a unitary transformation would preserve the essential self-adjointness properties).

\section{Appendix B. Spectrum of the operator $\overline{\hat{U}}_{k \omega}$. Alternative Proof.}

For simplicity, we take into account only the case of vanishing magnetic charge $q_{m}=0$. We mean to make use of theorem 3 in [38. The multiplication operator by

$$
R W \mathbb{V}_{\omega} W^{*} R^{*}=a \omega \frac{\sin (\theta)}{\sqrt{\Delta_{\theta}}}\left(\begin{array}{ll}
0 & 1 \\
1 & 0
\end{array}\right)
$$

is a bounded perturbation of the operator $R W \overline{\hat{U}}_{k} W^{*} R^{*}$. Then we consider first the spectrum of the latter operator. In order to apply the theory described in [38 it is useful to rewrite the (unperturbed) differential system $R W \mathbb{U}_{k} W^{*} R^{*} \Theta=\lambda \Theta$ as follows:

$$
\Theta^{\prime}=-\frac{k \Xi}{\Delta_{\theta} \sin (\theta)}\left(\begin{array}{cc}
1 & \frac{1}{k \Xi}\left[(\mu a \cos (\theta)+\lambda) \sqrt{\Delta_{\theta}} \sin (\theta)\right] \\
\frac{1}{k \Xi}\left[(\mu a \cos (\theta)-\lambda) \sqrt{\Delta_{\theta}} \sin (\theta)\right] & -1
\end{array}\right) \Theta \Leftrightarrow \Theta^{\prime}=: \mathcal{U} \Theta
$$


where the prime stays for the derivative with respect to $\theta$. (Notice that, by comparing (1.4) of [38] with our operator, we have to shift $\lambda \mapsto-\lambda$ in (1.1) of [38). According to the theory in [38, we are in the diagonally dominant case. Moreover, in order to face with our problem, which displays two singular endpoints, we refer to Remark (3), pp. 119-120, of [38, according to which if discrete spectrum criteria are satisfied at both endpoints, then the spectrum is discrete. Let us introduce two auxiliary selfadjoint operators:

$$
\begin{aligned}
& D\left(\hat{\mathbb{U}}_{0}\right)=\left\{\Theta \in L^{2}\left((0, c), \frac{d \theta}{\sqrt{\Delta_{\theta}}}\right)^{2} ; \Theta \text { is locally absolutely continuous; } B(\Theta)=0 ; \hat{\mathbb{U}}_{0} \Theta \in L^{2}\left((0, c), \frac{d \theta}{\sqrt{\Delta_{\theta}}}\right)^{2}\right\}, \\
& \hat{\mathbb{U}}_{0} \Theta=R W \mathbb{U}_{k} W^{*} R^{*} \Theta, \quad \Theta \in D\left(\hat{\mathbb{U}}_{0}\right) ; \\
& D\left(\hat{\mathbb{U}}_{\pi}\right)=\left\{\Theta \in L^{2}\left((c, \pi), \frac{d \theta}{\sqrt{\Delta_{\theta}}}\right)^{2} ; \Theta \text { is locally absolutely continuous; } B(\Theta)=0 ; \hat{\mathbb{U}}_{\pi} \Theta \in L^{2}\left((c, \pi), \frac{d \theta}{\sqrt{\Delta_{\theta}}}\right)^{2}\right\}, \\
& \hat{\mathbb{U}}_{\pi} \Theta=R W \mathbb{U}_{k} W^{*} R^{*} \Theta, \quad \Theta \in D\left(\hat{\mathbb{U}}_{\pi}\right) .
\end{aligned}
$$

$0<c<\pi$ is an arbitrary (regular) point at which the boundary condition $B(\Theta)=\sin (\beta) \Theta_{1}(c)+\cos (\beta) \Theta_{2}(c)=0$, with $\beta \in[0, \pi)$, is imposed for both.

According to the notation in [38, we have $p(\theta)=-\frac{k \Xi}{\Delta_{\theta} \sin (\theta)}, p_{1}(\theta)=p_{2}(\theta)=-\mu a \frac{\cos (\theta)}{\sqrt{\Delta_{\theta}}} \in L^{1}(0, \pi)$, and moreover $\alpha_{1}(\theta)=\alpha_{2}(\theta)=\frac{1}{\sqrt{\Delta_{\theta}}} \in L^{1}(0, \pi)$. Then $\frac{\alpha_{k}(\theta)}{p(\theta)}=-\sqrt{\Delta_{\theta}} \sin (\theta) \frac{1}{k \Xi}:=\alpha_{k 3}$, with $k=1,2$; furthermore, $a_{1}=0=a_{2}$ and then $q_{k}(\theta)=p_{k}(\theta)$, and $\frac{q_{k}(\theta)}{p(\theta)}=q_{k 3}(\theta)$. According to the definitions in [38] (p.102), $\alpha_{1}(\theta)=\alpha_{2}(\theta)$ and $p_{k}(\theta)$ are short range. As a consequence, we get $\Gamma_{1}=\Gamma_{2}=0$ and $q_{k 1}=0=q_{k 2}$ (cf. 38, p. 112). Then in the notation of $[38$, p. 112, we obtain

$$
D_{1}(\theta)=\left(\begin{array}{cc}
1 & 0 \\
0 & -1
\end{array}\right)
$$

and

$$
D_{3}(\theta)=\left(\begin{array}{cc}
0 & q_{23}-\lambda \alpha_{23} \\
-q_{13}+\lambda \alpha_{13} & 0
\end{array}\right)
$$

whereas the matrix $D_{2}(\theta)$ in our case is zero. Moreover we obtain

$$
\mu_{0}(\theta, \lambda)=1
$$

and, as a consequence, the matrix $\mathcal{S}$ defined in [38, p. 113, is the identity matrix in our case. One also obtains

$$
\begin{aligned}
E(\theta, \lambda) & =\exp \left(\int_{c}^{\theta} d t p(t)\right) \\
& =\left.\exp \left[-\frac{k}{2}\left(\frac{a}{l} \log \frac{l+a \cos (t)}{l-a \cos (t)}-\log \frac{1+\cos (t)}{1-\cos (t)}\right)\right]\right|_{c} ^{\theta},
\end{aligned}
$$

and can define the matrix

$$
\Omega(\theta, \lambda)=\left(\begin{array}{cc}
E(\theta, \lambda) & 0 \\
0 & \frac{1}{E(\theta, \lambda)}
\end{array}\right)
$$

The matrix $B(\theta)$ defined in [38, p. 113, is zero. The matrix $C(\theta)$ in our case coincides with $p(\theta) D_{3}(\theta)$ and is absolutely integrable. The matrix $G$ defined at p. 113 of 38 is identically zero in our case. It is then easy to show that the hypotheses of theorem 3, p.113, of [38] are satisfied. The limit point case criterion given in theorem 3 of 38 is easily verified both at $\theta=0$ and at $\theta=\pi$ due to (B.7), confirming the analysis carried out in the previous sections. Moreover, the criteria $\int_{c}^{0} d t|p(t)|=\infty$ and $\int_{c}^{\pi} d t|p(t)|=\infty$ are both satisfied, and then both $\hat{\mathbb{U}}_{0}$ and $\hat{\mathbb{U}}_{\pi}$ have discrete spectra. As a consequence, $\overline{\mathbb{U}}_{k}$ has discrete spectrum. As a consequence of theorem 10.8 in 21], we can also conclude that the spectrum is simple.

The bounded perturbation $W \mathbb{V}_{\omega} W^{*}$ affects the previous analysis in the following sense: the discrete eigenvalues $\lambda$ get an analytic dependence on $\omega$. Indeed, $W \mathbb{V}_{\omega} W^{*}$ is infinitesimally small with respect to $W \overline{\mathbb{U}}_{k} W^{*}[25$ ] and then $W \mathbb{U}_{k \omega} W^{*}$ defines an analytical family of type (A) according to Kato's definition [26, 27]. See in particular [27, p.16. 


\section{Appendix C. Discrete spectrum of $\hat{h}_{\infty}$. Alternative proof}

We refer to the results contained in [38]. It is useful to rewrite (6.9) in the following form:

$$
X^{\prime}=\left(\begin{array}{cc}
p(r) & -\omega \alpha(r)+p_{2}(r) \\
\omega \alpha(r)-p_{1}(r) & -p(r)
\end{array}\right) X,
$$

where $p(r):=\frac{\lambda_{k ; j}}{\sqrt{\Delta_{r}}}, \alpha(r):=\frac{r^{2}+a^{2}}{\Delta_{r}}, p_{1}(r):=\frac{1}{\Delta_{r}}\left(a \Xi k+e q_{e} r+\mu r \sqrt{\Delta_{r}}\right)$ and $p_{2}(r):=\frac{1}{\Delta_{r}}\left(a \Xi k+e q_{e} r-\mu r \sqrt{\Delta_{r}}\right)$.

We study the spectral properties of $\hat{h}_{\infty}$. In order to apply the theory exposed in [38, theorem 1, pp. 102-103, we put $p_{11}(r):=\mu r \frac{1}{\sqrt{\Delta_{r}}}$, which is positive as far as $\mu>0$ in the physical case, and $p_{21}(r):=-\mu r \frac{1}{\sqrt{\Delta_{r}}}$. As a consequence, being by assumption $p_{1}(r)=p_{11}(r)+p_{12}(r)$ and analogously $p_{2}(r)=p_{21}(r)+p_{22}(r)$, one finds $p_{12}(r)=\frac{1}{\Delta_{r}}\left(a \Xi k+e q_{e} r\right)$ and $p_{22}(r)=\frac{1}{\Delta_{r}}\left(a \Xi k+e q_{e} r\right)$; furthermore, one obtains $Q(r):=\sqrt{-p_{11}(r) p_{21}(r)}=$ $p_{11}(r)$. Moreover, one gets $\alpha_{1}(r)=\alpha_{2}(r)=\alpha(r)$, and then $\frac{-\alpha_{k}(r)}{p_{k 1}(r)}=r_{k 3}(r)$, with $k=1,2$, where $Q(r) r_{k 3}(r) \in$ $L^{1}\left[r_{0}, \infty\right)$ (note that there is a misprint in [38], p. 102, regarding this condition: $\frac{-\alpha_{k}(r)}{p_{k 2}(r)}$ is indicated in place of $\left.\frac{-\alpha_{k}(r)}{p_{k 1}(r)}\right)$. The latter property means that $Q(r) r_{k 3}(r)$ is short range (cf. [38, p. 102) (note that $Q(r) \sim \frac{\mu l}{r}$ and $\frac{-\alpha_{k}(r)}{p_{k 1}(r)} \sim(-)^{k} \frac{l}{\mu} \frac{1}{r}$ as $\left.r \rightarrow \infty\right)$. As to the ratio $\frac{-p_{k 2}(r)}{p_{k 1}(r)}$, we find that both the terms are short range, and then we can put $\frac{-p_{k 2}(r)}{p_{k 1}(r)}=s_{k 3}(r)$. In our case we get $r_{k 1}(r)=0=r_{k 2}(r)$ and $s_{k 1}(r)=0=s_{k 2}(r)$. The function $\bar{\Delta}:=\left(p_{11}^{-1}(r) p_{11}^{\prime}(r)-p_{21}^{-1}(r) p_{21}^{\prime}(r)-p(r)\right) \frac{1}{Q(r)}$ (cf. [38], p. 103) is such that $\bar{\Delta}=-\frac{\lambda_{k ; j}}{\mu r}=\bar{\Delta}_{3}$, where $\bar{\Delta}_{3} Q(r) \in L^{1}\left[r_{0}, \infty\right)$ and then is short range.

The function $\mu_{0}(r, \omega)$ in our case is 1 and then the function $E(r, \omega)$ defined at p. 103 of [38] is

$$
E(r, \omega)=\exp \left(\int_{r_{0}}^{r} d t Q(t)\right)
$$

The condition (1.5) at p. 103 of of [38, which is necessary and sufficient in order to get the limit point case at infinity, in our case becomes:

$$
\int_{r_{0}}^{\infty} d t\left(E^{2}(r, 0)+E^{-2}(r, 0)\right)(2 \alpha(r))=\infty ;
$$

being $E^{ \pm 2}(r, 0) \sim r^{ \pm 2 \mu l}$ and $\alpha(r) \sim \frac{l^{2}}{r^{2}}$ for $r \rightarrow \infty$, one obtains the same conditions we found in our study of the essential selfadjointness of the radial Hamiltonian. Moreover, when the limit point case is implemented, the condition

$$
\int_{r_{0}}^{\infty} d t Q(t)=\infty
$$

is sufficient in order to get a discrete spectrum, and this condition is easily verified in our case. Then the spectrum of $\hat{h}_{\infty}$ is discrete. Note that, if the limit circle case occurs at infinity, the spectrum is still discrete (cf. theorem 7.11 in [21]).

\section{Note added}

After this work was completed, we were made aware of Ref. [39]. Therein a result about essential selfadjointness of $\hat{H}$ which is analogous to theorem 1 is stated for the Kerr-Newman case (cf. theorem 2.1 therein).

\section{REFERENCES}

[1] Callan, C.G. and Maldacena J.M.: Black holes in string theory. Prepared for ICTP Summer School in High-energy Physics and Cosmology, Trieste, Italy, 10 Jun - 26 Jul 1996. In Trieste 1996, High-energy Physics and Cosmology. pp. 1-65.

[2] Chrusciel, P.T.: 'No hair' theorems: Folklore, conjectures, results. Contemp. Math. 170, 23-49 (1994).

Heusler, M.: No-hair theorems and black holes with hair. Helv. Phys. Acta 69, 501-528 (1996).

[3] Damour, T.: Klein paradox and vacuum polarization. In: Ruffini, R. (ed.) Proceedings of the first Marcel Grossmann Meeting on General Relativity. Proceedings, Trieste, 1975. Amsterdam: North-Holland, 1977, pp. 459-482.

Deruelle, N.: Classical and quantum states in black hole physics. In: Ruffini, R. (ed.) Proceedings of the first Marcel Grossmann Meeting on General Relativity. Proceedings, Trieste, 1975. Amsterdam: North-Holland, 1977, pp. 483-498. 
Soffel, M., Müller, B. and Greiner, W.: Particles in a Stationary Spherically Symmetric Gravitational Field. J. Phys. A10, 551-561 (1977).

[4] Belgiorno, F. and Martellini, M.: Quantum properties of the electron field in Kerr-Newman black hole manifolds. Phys. Lett. B453, 17-22 (1999).

[5] Belgiorno, F. and Cacciatori, S.L.: Quantum Effects for the Dirac Field In Reissner-Nordström-AdS Black Hole Background. Class. Quant. Grav. 25, 105013 (2008).

[6] Hawking, S.W. and Page, D.N.: Thermodynamics of Black Holes in Anti-de Sitter Space. Commun. Math. Phys. 87, 577-588 (1983).

[7] Wald, R.: Dynamics in nonglobally hyperbolic, static space-times. J. Math. Phys. 21, 2802-2805 (1980). Horowitz, G.T. and Marolf, D.: Quantum probes of space-time singularities. Phys. Rev. D52, 5670-5677 (1995).

[8] Kamran, N. and Mclenaghan, R.G.: Separation Of Variables And Symmetry Operators For The Neutrino And Dirac Equations In The Space-Times Admitting A Two Parameter Abelian Orthogonally Transitive Isometry Group And A Pair Of Shearfree Geodesic Null Congruences. J. Math. Phys. 25, 1019-1027 (1984).

[9] Finster, F., Kamran, N., Smoller, J. and Yau, S.T.: The long-time dynamics of Dirac particles in the Kerr-Newman black hole geometry. Adv. Theor. Math. Phys. 7, 25-52 (2003)

[10] Finster, F., Smoller, J. and Yau, S.T.: Non-existence of time-periodic solutions of the Dirac equation in a Reissner-Nordstroem black hole background. J. Math. Phys. 41, 2173-2194 (2000).

[11] Finster, F., Kamran, N., Smoller, J. and Yau, S.T.: Non-existence of time-periodic solutions of the Dirac equation in an axisymmetric black hole geometry. Comm. Pure Appl. Math. 53, 902-929 (2000).

[12] Winklmeier, M. and Yamada, O.: Spectral analysis of radial Dirac operators in the Kerr-Newman metric and its applications to time-periodic solutions. J. Math. Phys. 47, 102503 (2006).

[13] Schmid, H.: Bound state solutions of the Dirac equation in the extreme Kerr geometry. Math. Nachr. 274-275, 117 (2004). Batic, D. and Schmid, H.: The Dirac propagator in the extreme Kerr metric. J. Phys. A40, 13443-13452 (2007).

[14] Batic, D. and Schmid, H.:The Dirac propagator in the Kerr-Newman metric. Prog.Theor.Phys. 116, 517-544 (2006).

[15] Häfner, D. and Nicolas, J.-P.: Scattering of massless Dirac fields by a Kerr black hole. Rev. Math. Phys. 16, 29-123 (2004).

[16] Dafermos, M.: On 'time periodic' black hole solutions to certain spherically symmetric Einstein matter systems. Commun. Math. Phys. 238, 411-427 (2003).

[17] Caldarelli, M.M., Cognola, G. and Klemm, D.: Thermodynamics of Kerr-Newman-AdS black holes and conformal field theories. Class. Quant. Grav. 17 399-420 (2000).

[18] Weidmann, J.: Linear Operators in Hilbert Spaces. Graduate Texts in Mathematics 68. Berlin: Springer-Verlag, 1980.

[19] Winklmeier, M.: The Angular Part of the Dirac Equation in the Kerr-Newman Metric: Estimates for the Eigenvalues. Ph.D thesis (2005).

[20] Mlak, W: Hilbert Spaces and Operator Theory. Mathematics and Its Application (East European Series) Vol. 51. Dordrecht: Kluwer Academic Publishers, 1991.

[21] Weidmann, J.: Spectral Theory of Ordinary Differential Operators. Lecture Notes in Mathematics 1258. Berlin: SpringerVerlag, 1987.

[22] Hsieh, P.-F., Sibuya, Y.: Basic Theory of Ordinary Differential Equations. Berlin: Springer, 1999.

[23] Walter, W.: Ordinary Differential Equations. Graduate Texts in Mathematics 182. Berlin: Springer, 1998.

[24] Batic, D., Winklmeier, M. and Schmid H.: On the eigenvalues of the Chandrasekhar-Page angular equation. J. Math. Phys. 46, 012504 (2005).

[25] Reed, M. and Simon, B.: Methods of modern mathematical physics. Vol II: Fourier analysis, self-adjointness. San Diego: Academic Press, 1975.

[26] Kato, T.: Perturbation theory for linear operators. Classics in mathematics. Berlin: Springer-Verlag, 1995.

[27] Reed, M. and Simon, B.: Methods of modern mathematical physics. Vol IV: Analysis of operators. San Diego: Academic Press, 1978.

[28] Schmidt, K.M.: Dense point spectrum and absolutely continous spectrum in spherically symmetric Dirac operators. Forum Math. 7, 459-475 (1995).

[29] Weidmann, J.: Oszillationsmethoden für Systeme gewönlicher Differentialgleichungen. Math. Z. 119, 349-373 (1971).

[30] Hinton, D.B. and Shaw, J.K.: Absolutely continuous spectra of Dirac systems with long range, short range and oscillating potentials. Quart. J. Math. Oxford 36, 183-213 (1985).

[31] Weidmann, J.: Absolut stetiges Spektrum bei Sturm-Liouville-Operatoren und Dirac-Systemen. Math. Z. 180, 423-427 (1982).

[32] Eastham, M.S.P.: The Asymptotic Solution Of Linear Differential Systems. Applications of the Levinson theorem. London Mathematical Society Monographs New Series 4. Oxford Science Publications. Oxford: Clarendon Press, 1989.

[33] Schwinger, J.: Magnetic charge and quantum field theory. Phys. Rev. 144, 1087-1093 (1966).

[34] Blagojević, M. and Senjanović, P.: The quantum field theory of electric and magnetic charge. Phys. Rep. 157, 233-346 (1988); Schwinger, J.: Magnetic charge and the charge quantization condition. Phys. Rev. D15, 3105-3111 (1975);

Nesterov, A.I. and de la Cruz, F.A.: Infinite dimensional representations of the rotation group and Dirac monopole problem. J. Math. Phys. 49, 013505 (2008).

[35] Wu, T.T. and Yang, C.N.: Dirac monopole without strings: monopole harmonics. Nucl. Phys. B107, 365-380 (1976).

[36] Ghosh, R.K., Pal, P.B.: A non-singular potential for the Dirac monopole. Phys. Lett. B551, 387-390 (2003).

[37] Barut, A.O. and Wilson, R.: On the Connection between Dyons, Vortices, and Aharonov-Bohm Effect. Ann. Phys. 164, 223-232 (1985). 
[38] Hinton, D.B. and Shaw, J.K.: Dirac systems with discrete spectra. Can. J. Math. XXXIX, 100-122 (1987).

[39] Winklmeier, M. and Yamada, O.: On the local energy decay of solutions of the Dirac equation in the non-extreme Kerr-Newman metric. I. e-Print: arXiv:0802.0402 1 (2008).

Dipartimento di Fisica, Università degli Studi di Milano, Via Celoria 16, 20133 Milano, Italy

E-mail address: belgiorno@mi.infn.it

Dipartimento di Fisica, Università degli Studi dell'Insubria, Via Valleggio 11, 22100 Como, Italy

E-mail address: sergio.cacciatori@uninsubria.it 